\title{
Grapevine microRNAs responsive to exogenous gibberellin
}

\author{
Jian Han ${ }^{1}$, Jinggui Fang ${ }^{1 *}$, Chen Wang ${ }^{1 *}$, Yanlei Yin ${ }^{2}$, Xin Sun ${ }^{1}$, Xiangpeng Leng ${ }^{1}$ and Changnian Song ${ }^{1}$
}

\begin{abstract}
Background: MicroRNAs (miRNAs), involving in various biological and metabolic processes, have been discovered and analyzed in quite a number of plants species, such as Arabidopsis, rice and other plants. However, there have been few reports about grapevine miRNAs in response to gibberelline $\left(\mathrm{GA}_{3}\right)$.

Results: Solexa technology was used to sequence small RNA libraries constructed from grapevine berries treated with $\mathrm{GA}_{3}$ and the control. A total of 122 known and 90 novel grapevine miRNAs (Vvi-miRNAs) were identified. Totally, 137 ones were found to be clearly responsive to $\mathrm{GA}_{3}$, among which 58 were down-regulated, 51 were up-regulated, 21 could only be detected in the control, and seven were only detected in the treatment. Subsequently, we found that 28 of them were differentially regulated by $\mathrm{GA}_{3}$, with 12 conserved and 16 novel Vvi-miRNAs, based on the analysis of qRT-PCR essays. There existed some consistency in expression levels of $\mathrm{GA}_{3}$-responsive Vvi-miRNAs between high throughput sequencing and qRT-PCR essays. In addition, 117 target genes for 29 novel miRNAs were predicted.
\end{abstract}

Conclusions: Deep sequencing of short RNAs from grapevine berries treated with GA3 and the control identified 137 $\mathrm{GA}_{3}$-responsive miRNAs, among which 28 exhibited different expression profiles of response to $\mathrm{GA}_{3}$ in the diverse developmental stages of grapevine berries. These identified Vvi-miRNAs might be involved in the grapevine berry development and response to environmental stresses.

Keywords: Grapevine, Berry, microRNAs, Exogenous gibberellin, High throughput sequencing

\section{Background}

MicroRNAs (miRNAs) are endogenous gene regulators distributed widely in plant genomes, and they play important roles in plant growth, development, signal transduction and response to environmental stimuli [1-5]. Identification of miRNAs is a key step in gaining insight into sRNA-based regulatory functions with many conserved miRNAs having been identified by traditional sequencing approaches such as the Sanger sequencing method [6]. However, most species-specific or tissue-specific miRNAs are hard to be detected probably because of their low accumulation and/ or insufficient stringency of the sequencing approach [7-9]. The advent of new sequencing technologies could make it possible to mine even species-/tissue- specific miRNAs with low abundance, and they havebeen successfully used on Arabidopsis thaliana, Oryza sativa, Poplus tricocarpa, Medicago tuncatula, Gossypiumhirsutum, Zea

\footnotetext{
* Correspondence: fanggg@njau.edu.cn; wangchen@njau.edu.cn

${ }^{1}$ College of Horticulture, Nanjing Agricultural University, Nanjing 210095, China

Full list of author information is available at the end of the article
}

mays, Arachis hypogaea L., Solanumlycopersicum, Citrus trifoliate, Vitis vinifera and Vitis amurensis Rupr. [10-21].

Grapevine (Vitis vinifera L.) is one of the most economically important fruit crops worldwide and has nutritional and processing properties [22]. In recent years, sequencing of small RNA libraries from different grapevine cultivars or tissues has severally been reported where a large number of Vvi-miRNAs were identified [7,18-21]. Despite this, there are no reports on the study of response of Vvi-miRNAs to phytohormones. Phytohormones are important endogenous signals and regulators involved in plant growth and development [23], and they are classified into auxins, gibberellins $\left(\mathrm{GA}_{3}\right)$, cytokinins, abscisic acid(ABA) and ethylene. All of these phytohormones act at low concentrations to regulate different aspects of plant growth and development to varying degrees [23-25]. Among the hormones, $\mathrm{GA}_{3}$ play significant regulatoryroles in early berry expansion, berry set and berry ripening [26,27]. Till now, it is still unclear on how $\mathrm{GA}_{3}$ participates in the regulation of the complicated developmental processes of grapevine berry.
C Biomed Central

(c) 2014 Han et al.; licensee BioMed Central Ltd. This is an Open Access article distributed under the terms of the Creative Commons Attribution License (http://creativecommons.org/licenses/by/2.0), which permits unrestricted use, distribution, and reproduction in any medium, provided the original work is properly credited. 
It has been reported that phytohormone signaling pathways can be effectively controlled by modulation of positive and negative regulators during plant growth and development [4]. Among the modulators of phytohormones, miRNA was recently found to be a new growth regulator involved in phytohormone signaling [28-30], with several studies showing the interactions between miRNAs and phytohormones in various plant responses. For instance, $\mathrm{GA}_{3}$ modulates the expression of miR159, while miR159 regulates the development of Arabidopsis anthers and seeds by cleaving the GAMYB gene, during Arabidopsis anther development [28] and seed germination [29]. In strawberry, miR159 interacts with GAMYB during the course of receptacle development, and both of them act in a joint fashion to respond, in part, to changes in endogenous $\mathrm{GA}_{3}$ levels [30]. These studies strongly demonstrated that miRNAs are involved in the $\mathrm{GA}_{3}$ signaling process.

To account for the roles of miRNAs in response to $\mathrm{GA}_{3}$ during grapevine berry development, we constructed two small RNA libraries from mixed tissue samples of grapevine berries sprayed with $\mathrm{GA}_{3}$ (treatment) and with water (the control). The grapevine cultivar used for this study is 'Summer Black' (hybrid of $V$. vinifera $\times V$. labrusca), an elite table grapevine cultivar native to Japan. After highthroughput sequencing, we identified a number of conserved and non-conserved Vvi-miRNAs responsive to $\mathrm{GA}_{3}$, and we further analyzed their potential role in mediation of GA3-induced regulation of grapevine berry growth and development. Further, qRT-PCR was utilized to analyze the expression of Vvi-miRNAs in different development stages of the grapevine berries subjected to exogenous $\mathrm{GA}_{3}$ and in exogenous $\mathrm{GA}_{3}$-free berries. Lastly, an attempt to elucidate the regulatory functions of Vvi-miRNAs being responsive to $\mathrm{GA}_{3}$ during grapevine berry development was done.

\section{Results}

Characterization of the Vvi-miRNAs from deep sequencing of grapevine sRNA libraries

To identify $\mathrm{GA}_{3}$-responsive miRNAs in grapevine berries, two small RNA libraries from grapevine berries treated with $\mathrm{GA}_{3}\left(\mathrm{GA}_{3}\right.$ treatment) and sprayed with water (the control) were constructed. Solexa, a high throughput sequencing technology, was employed to sequence these libraries, leading to a generation of 16,231,320 and 16,486,660 clean reads from $\mathrm{GA}_{3}$ treatment and the control libraries, respectively. All these clean reads were those from removal of adaptor, insert, polyA, and RNAs shorter than 18nt in length (Table 1). About 4,265,160 ( $\mathrm{GA}_{3}$ treatment) and $4,326,915$ (the control) clear reads could be mapped to the grapevine genome published in 2007 [31], and miRNA, tRNA, siRNA, snRNA, snoRNA, rRNA, repeat regions, exon and intron RNA reads were annotated. In addition, 9,075,238 and 9,207,588 un-annotated reads were used for prediction of new Vvi-miRNAs in $\mathrm{GA}_{3}$ treatment and the control grapevine berries, respectively (Table 1 ).

The size distribution of all sRNAs was found to be uneven ranging from $18 \mathrm{nt}$ to $30 \mathrm{nt}$ long, with the majority being 19-25nt long (Figure 1). The sRNAs of $21 \mathrm{nt}$ and 24nt formed two major classes, occupying $38.35 \%$ and $32.06 \%$ (Figure 1) of the total, respectively, an observation which is in agreement with some previous reports in grapevine and tomato $[11,16,21,31]$, but contrasts to those reported in Arabidopsis, rice and peanut [32-35]. The two main peaks of sRNAs in this study were 24 and 21 nt long, while the most common sRNAs in Taxus chinensis [35] and Citrus trifoliate [17] were those with $21 \mathrm{nt}$ in length. These cases suggested that some differences might exist in the sRNA biogenesis pathways in various plants. In addition, analysis of the first nucleotide of 18-25nt long sRNAs

Table 1 Distribution of small RNAs among different categories in control and $\mathrm{GA}_{3}$ Treated grapevine berries

\begin{tabular}{|c|c|c|c|c|c|c|c|c|}
\hline \multirow[t]{2}{*}{ Category } & \multicolumn{4}{|c|}{ Control } & \multicolumn{4}{|c|}{ GA3 treatment } \\
\hline & Unique & Percent (\%) & Redundant & Percent (\%) & Unique & Percent (\%) & Redundant & Percent (\%) \\
\hline Exon_antisense & 88740 & $2.28 \%$ & 232050 & $1.41 \%$ & 67193 & $1.40 \%$ & 157256 & $0.98 \%$ \\
\hline Exon_sense & 136907 & $3.52 \%$ & 449869 & $2.73 \%$ & 130285 & $2.71 \%$ & 289348 & $1.80 \%$ \\
\hline Intron_antisense & 35335 & $0.91 \%$ & 72189 & $0.44 \%$ & 32881 & $0.68 \%$ & 52624 & $0.33 \%$ \\
\hline Intron_sense & 41391 & $1.06 \%$ & 106364 & $0.65 \%$ & 39164 & $0.81 \%$ & 72984 & $0.46 \%$ \\
\hline miRNA & 1724 & $0.04 \%$ & 683061 & $4.14 \%$ & 1726 & $0.04 \%$ & 812099 & $5.07 \%$ \\
\hline rRNA & 51658 & $1.33 \%$ & 1099955 & $6.67 \%$ & 72149 & $1.50 \%$ & 1108756 & $6.92 \%$ \\
\hline Repeat & 15219 & $0.39 \%$ & 27556 & $0.17 \%$ & 12601 & $0.26 \%$ & 22740 & $0.14 \%$ \\
\hline snRNA & 4408 & $0.11 \%$ & 19905 & $0.12 \%$ & 4553 & $0.09 \%$ & 19873 & $0.12 \%$ \\
\hline snoRNA & 1780 & $0.05 \%$ & 5172 & $0.03 \%$ & 1895 & $0.04 \%$ & 6403 & $0.04 \%$ \\
\hline tRNA & 13078 & $0.34 \%$ & 1285951 & $7.80 \%$ & 20052 & $0.42 \%$ & 1210840 & $7.55 \%$ \\
\hline unann & 3501164 & $89.97 \%$ & 9207588 & $55.85 \%$ & 4190436 & $87.17 \%$ & 9075238 & $56.60 \%$ \\
\hline Mapping to genome & 838267 & $21.54 \%$ & 43326915 & $26.24 \%$ & 765402 & $15.92 \%$ & 4265160 & $26.60 \%$ \\
\hline Total & 3891404 & $100.00 \%$ & 16486660 & $100.00 \%$ & 4807290 & $100 \%$ & 16033016 & $100 \%$ \\
\hline
\end{tabular}




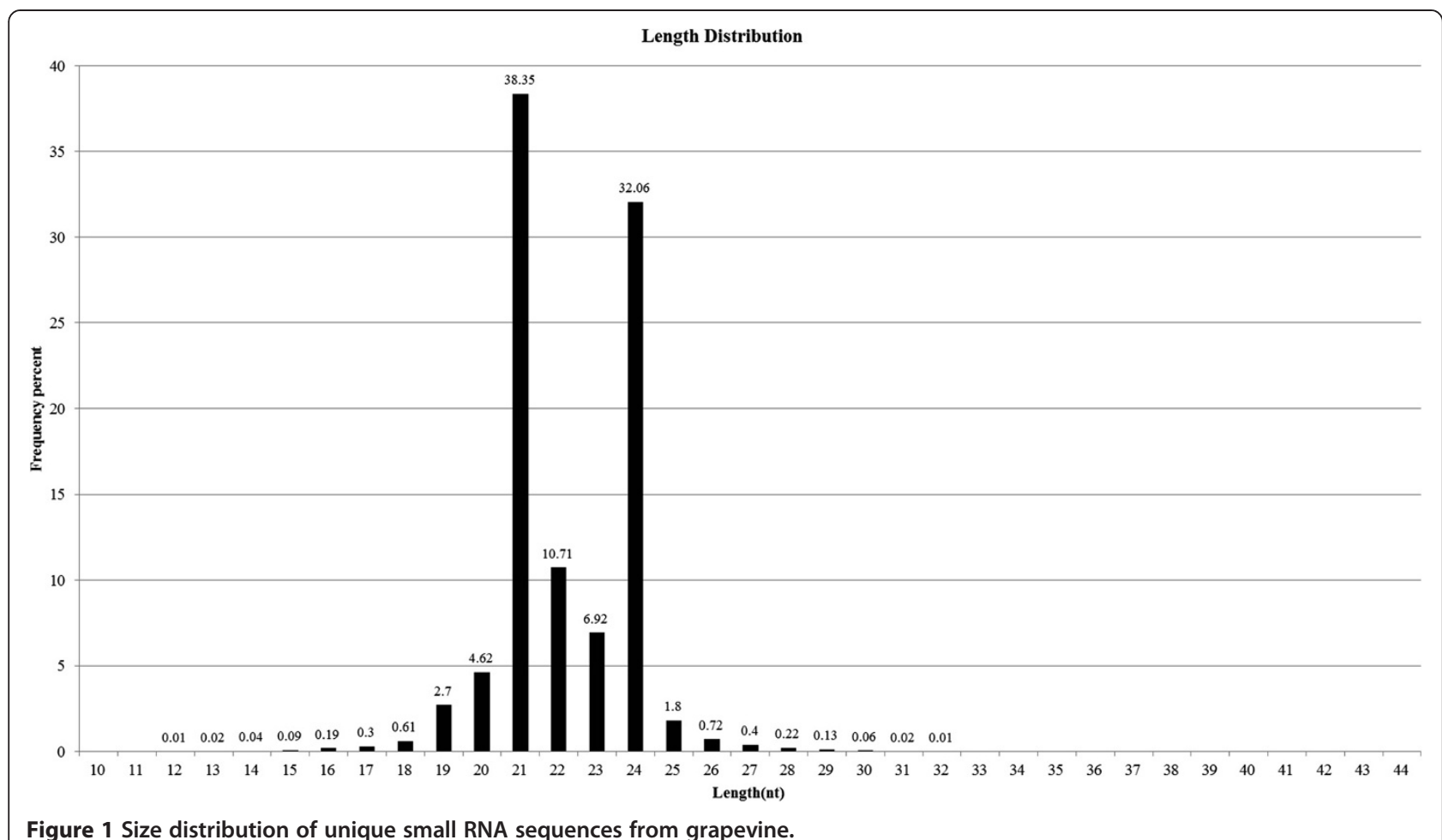

indicated that many sRNAs started with a uridine $(\mathrm{U})$ at their 5 '-ends and most of them are $21 \mathrm{nt}$ and $22 \mathrm{nt}$ long, with the former being most outstanding in number (Figure 2). Similar to other plants, most miRNAs here were those with 21 and 22 nt in length and they also begin with a 5 'uridine, which is one of the important characteristic features of miRNAs $[10,11,36]$.

High throughput sequencing can verify a large number of known miRNAs together with the identification of novel specific miRNAs even with the low abundance in organisms. From two sRNA libraries in this study, we first searched for known Vvi-miRNAs by comparing our libraries with known miRNAs from other plant species in miRBase 19.0 (http://www.mirbase.org/). A total of 122 known Vvi-miRNAs were sequenced both in the control and $\mathrm{GA}_{3}$-treatment libraries, and they belonged to 27 conserved miRNA families according to a comparative genomics-based analysis in different plant species [37]. Eighteen of the 27 Vvi-miRNA families contained many members (Table 2), with four families (Vvi-miR169, VvimiR156, Vvi-miR166, Vvi-miR171 and Vvi-miR399) possessing 19, 8, 7, 8, and 7 members, respectively. Another nine Vvi-miRNA families (Vvi-miR162, Vvi-miR168, Vvi-miR390, Vvi-miR397, Vvi-miR408, Vvi-miR477, Vvi-miR479, Vvi-miR482, and Vvi-miR828) had only one member each. Among the known Vvi-miRNAs, the Vvi-miR166 family had the most abundant reads accounting for $63.2 \%$ of all the conserved miRNA reads. In this family, the number of Vvi-miR166h reads was over 500,000 in the two libraries, followed

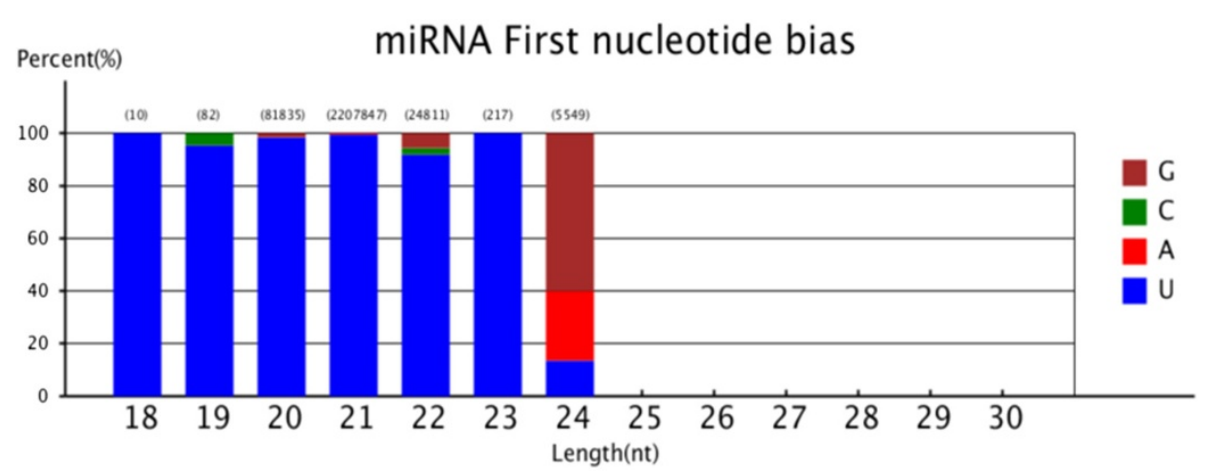

Figure 2 First nucleotide bias of 18-30nt sRNA tags. 
Table 2 Conserved Vv-miRNAs identified and normalized counts (NC) in control and $\mathrm{GA}_{3}$ treated grapevine berries

\begin{tabular}{|c|c|c|c|c|c|c|}
\hline \multicolumn{3}{|c|}{ Control } & \multicolumn{3}{|c|}{ GA3treatment } & \multirow[t]{2}{*}{ Identity } \\
\hline miRNA ID & Sequences & NC & miRNA ID & Sequences & NC & \\
\hline Vvi-miR156a & TTGACAGAAGAGAGGGAGCAC & 8.81 & vv-miR156a & TTGACAGAAGAGAGGGAGCAC & 513.79 & $\uparrow$ \\
\hline Vvi-miR156b & AACTGACAGAAGAGAGTGAGCAC & 2308.90 & vv-miR156b & AACTGACAGAAGAGAGTGAGCAC & 0.61 & $\downarrow$ \\
\hline Vvi-miR156c & TGACAGAAGAGAGTGAGCACAC & 2214.31 & vv-miR156c & TGACAGAAGAGAGTGAGCACAC & 0.17 & $\downarrow$ \\
\hline Vvi-miR156d & TGACAGAAGAGAGTGAGCAC & 2321.38 & vv-miR156d & TGACAGAAGAGAGTGAGCAC & 2331.59 & $\bullet$ \\
\hline Vvi-miR156e & TGACAGAGGAGAGTGAGCAC & 3.40 & vv-miR156e & TGACAGAGGAGAGTGAGCAC & 14.83 & $\uparrow$ \\
\hline Vvi-miR156f & CTGTTGACAGAAGATAGAGAGCAC & 5215.88 & vv-miR156f & CTGTTGACAGAAGATAGAGAGCAC & 3.93 & $\downarrow$ \\
\hline Vvi-miR156g & GCTCTCTAGACTTCTGTCATC & 5214.31 & vv-miR156g & GCTCTCTAGACTTCTGTCATC & 0.61 & $\downarrow$ \\
\hline \multirow[t]{2}{*}{ Vvi-miR156i } & TTGACAGAAGATAGAGAGCAC & 5214.31 & vv-miR156i & TTGACAGAAGATAGAGAGCAC & 6093.28 & $\uparrow$ \\
\hline & & 0.00 & vv-miR159b & CCTTGGAGTGAAGGGAGCT & 0.17 & $\Delta$ \\
\hline Vvi-miR159c & TाTGATTGAAGGGAGCTCTA & 74.61 & vv-miR159c & TTGGATTGAAGGGAGCTCTA & 582.11 & $\uparrow$ \\
\hline Vvi-miR160a & TCCTAGTTGGCATCAGAGGAG & 1.66 & vv-miR160a & TCCTAGTTGGCATCAGAGGAG & 0.09 & $\downarrow$ \\
\hline Vvi-miR160b & GCATGAGGGGAGTCAAGCAGG & 1.66 & vv-miR160b & GCATGAGGGGAGTCAAGCAGG & 4.19 & $\uparrow$ \\
\hline Vvi-miR160c & GCGTGCGAGGAGCCAAGCATA & 3.75 & vv-miR160c & GCGTGCGAGGAGCCAAGCATA & 4.54 & $\bullet$ \\
\hline Vvi-miR160d & TGCCTGGCTCCCTGTATGCCA & 4.71 & & & 0.00 & $\nabla$ \\
\hline \multirow[t]{2}{*}{ Vvi-miR160e } & GCGTATGAGGAGCCATGCATA & 4.71 & vv-miR160e & GCGTATGAGGAGCCATGCATA & 2.27 & $\downarrow$ \\
\hline & & 0.00 & vv-miR160f & TGCCTGGCTCCCTGTATGCCA & 43.98 & $\boldsymbol{\Delta}$ \\
\hline Vvi-miR162 & TCGATAAACCTCTGCATCCAG & 236.04 & vv-miR162 & TCGATAAACCTCTGCATCCAG & 231.76 & $\bullet$ \\
\hline Vvi-miR164a & TTGGAGAAGCAGGGCACGTGC & 43.02 & vv-miR164a & TTGGAGAAGCAGGGCACGTGC & 0.17 & $\downarrow$ \\
\hline Vvi-miR164c & TGGAGAAGCAGGGCACGTGCAT & 43.19 & vv-miR164c & TGGAGAAGCAGGGCACGTGCAT & 6.72 & $\downarrow$ \\
\hline Vvi-miR164d & TGGAGAAGCAGGGCACGTGCA & 43.02 & vv-miR164d & TGGAGAAGCAGGGCACGTGCA & 798.08 & $\uparrow$ \\
\hline Vvi-miR166a & TCTCGGACCAGGCTTCATTCCT & 762.04 & vv-miR166a & TCTCGGACCAGGCTTCATTCCT & 7.85 & $\downarrow$ \\
\hline Vvi-miR166b & TCGGACCAGGCTTCATTCCTC & 3280.28 & vv-miR166b & TCGGACCAGGCTTCATTCCTC & 15371.38 & $\uparrow$ \\
\hline Vvi-miR166c & TCGGACCAGGCTTCATTCCCC & 21467.36 & & & 0.00 & $\nabla$ \\
\hline Vvi-miR166d & GATTGTTGTCTGGCTCGAGGC & 21492.15 & vv-miR166d & GATTGTTGTCTGGCTCGAGGC & 1.05 & $\downarrow$ \\
\hline Vvi-miR166e & GGAATGTTGTCTGGCTCGAGG & 21467.36 & vv-miR166e & GGAATGTTGTCTGGCTCGAGG & 311.87 & $\downarrow$ \\
\hline Vvi-miR166f & GGAATGTTGGCTGGCTCGAGG & 21506.81 & vv-miR166f & GGAATGTTGGCTGGCTCGAGG & 18.24 & $\downarrow$ \\
\hline Vvi-miR166g & TTCGGACCAGGCTTCATTCCC & 21509.60 & vv-miR166g & TTCGGACCAGGCTTCATTCCC & 82.11 & $\downarrow$ \\
\hline Vvi-miR166h & TCGGACCAGGCTTCATTCCCC & 22171.73 & vv-miR166h & TCGGACCAGGCTTCATTCCCC & 22520.68 & $\bullet$ \\
\hline Vvi-miR167a & TGAAGCTGCCAGCATGATCTGG & 32.81 & vv-miR167a & TGAAGCTGCCAGCATGATCTGG & 11.78 & $\downarrow$ \\
\hline Vvi-miR167b & TGAAGCTGCCAGCATGATCTAAG & 616.06 & vv-miR167b & TGAAGCTGCCAGCATGATCTAAG & 38.48 & $\downarrow$ \\
\hline Vvi-miR167c & TGAAGCTGCCAGCATGATCTC & 134.12 & vv-miR167c & TGAAGCTGCCAGCATGATCTC & 1901.57 & $\uparrow$ \\
\hline Vvi-miR167d & TGAAGCTGCCAGCATGATCTAG & 424.87 & vv-miR167d & TGAAGCTGCCAGCATGATCTAG & 1.75 & $\downarrow$ \\
\hline Vvi-miR167e & TGAAGCTGCCAGCATGATCTA & 613.09 & vv-miR167e & TGAAGCTGCCAGCATGATCTA & 2349.30 & $\uparrow$ \\
\hline Vvi-miR168 & TCGCTTGGTGCAGGTCGGGAA & 2963.35 & vv-miR168 & TCGCTTGGTGCAGGTCGGGAA & 2654.62 & $\bullet$ \\
\hline Vvi-miR169a & CAGCCAAGGATGACTTGCCGG & 13.09 & & & 0.00 & $\nabla$ \\
\hline Vvi-miR169b & GGTCGAATTGAGCCAAGGATGG & 5.58 & vv-miR169b & GGTCGAATTGAGCCAAGGATGG & 0.35 & $\downarrow$ \\
\hline Vvi-miR169c & TCCGGCAAGTTGTCCTTGGCTAC & 13.09 & vv-miR169c & TCCGGCAAGTTGTCCTTGGCTAC & 0.70 & $\downarrow$ \\
\hline Vvi-miR169d & CAGCCAAGAATGATTTGCCGG & 35.25 & vv-miR169d & CAGCCAAGAATGATTTGCCGG & 105.50 & $\uparrow$ \\
\hline Vvi-miR169e & TAGCCAAGGATGACTTGCCT & 0.44 & & & 0.00 & $\nabla$ \\
\hline Vvi-miR169f & TGGGCAAGTTGTGTTTGGCTAC & 0.35 & vv-miR169f & TGGGCAAGTTGTGTTTGGCTAC & 0.26 & $\bullet$ \\
\hline Vvi-miR169g & CAGCCAAGGATGACTTGCCGA & 0.44 & vv-miR169g & CAGCCAAGGATGACTTGCCGA & 6.54 & $\uparrow$ \\
\hline Vvi-miR169h & TGAGCCAAGGATGGCTTGCCGT & 5.50 & vv-miR169h & TGAGCCAAGGATGGCTTGCCGT & 7.42 & $\bullet$ \\
\hline Vvi-miR169i & CTGGTCATGCACGGCTGGTTA & 1.83 & vv-miR169i & CTGGTCATGCACGGCTGGTTA & 0.09 & $\downarrow$ \\
\hline
\end{tabular}


Table 2 Conserved Vv-miRNAs identified and normalized counts (NC) in control and $\mathrm{GA}_{3}$ treated grapevine berries (Continued)

\begin{tabular}{|c|c|c|c|c|c|c|}
\hline Vvi-miR169j & CAGCCAAGGATGACTTGCCGG & 13.09 & & & 0.00 & $\boldsymbol{\nabla}$ \\
\hline Vvi-miR169k & AGCCAAGGATGACTTGCCGGA & 13.09 & vv-miR169k & AGCCAAGGATGACTTGCCGGA & 0.26 & $\downarrow$ \\
\hline Vvi-miR169l & TGAGCCAAGGATGACTTGCCGT & 58.12 & vv-miR169| & TGAGCCAAGGATGACTTGCCGT & 0.96 & $\downarrow$ \\
\hline Vvi-miR169m & TGAGCCAAGGATGACTTGCCG & 54.45 & & & 0.00 & $\boldsymbol{\nabla}$ \\
\hline Vvi-miR169n & AAGCATCTGAGGCTCTATTTC & 16.32 & vv-miR169n & AAGCATCTGAGGCTCTATTTC & 186.82 & $\uparrow$ \\
\hline Vvi-miR169o & TGAGCCAAGGATGACTTGCCG & 54.80 & vv-miR169o & TGAGCCAAGGATGACTTGCCG & 13.53 & $\downarrow$ \\
\hline Vvi-miR169p & GCAAGCATCCGAGGCTCTGT & 55.50 & vv-miR169p & GCAAGCATCCGAGGCTCTGT & 3.66 & $\downarrow$ \\
\hline Vvi-miR169q & TAGAGCCAAGGATGACTTGCCG & 16.40 & vv-miR169q & TAGAGCCAAGGATGACTTGCCG & 6.37 & $\downarrow$ \\
\hline Vvi-miR169r & TGAGTCAAGGATGACTTGCCGA & 4.71 & vv-miR169r & TGAGTCAAGGATGACTTGCCGA & 0.79 & $\downarrow$ \\
\hline Vvi-miR169s & CAGCCAAGGATGACTTGCCGG & 13.18 & & & 0.00 & $\boldsymbol{\nabla}$ \\
\hline Vvi-miR169t & GGCAAGTTGACTTGACTCAGT & 1.66 & vv-miR169t & GGCAAGTTGACTTGACTCAGT & 6.81 & $\uparrow$ \\
\hline Vvi-miR169u & GGCAAGTTGACTTGACTCTGT & 2.71 & vv-miR169u & GGCAAGTTGACTTGACTCTGT & 4.01 & $\bullet$ \\
\hline Vvi-miR169v & AAGCCAAGGATGAATTGCCGG & 4.36 & vv-miR169v & AAGCCAAGGATGAATTGCCGG & 2.62 & $\downarrow$ \\
\hline Vvi-miR169w & CAGCCAAGGATGACTTGCCGG & 13.18 & vv-miR169w & CAGCCAAGGATGACTTGCCGG & 12.39 & $\bullet$ \\
\hline Vvi-miR169x & TGAGTCAAGGATGACTTGCCGA & 0.52 & vv-miR169x & TGAGTCAAGGATGACTTGCCGA & 0.70 & $\bullet$ \\
\hline Vvi-miR171a & TGTTGGGACGGCTCAATCAAA & 4.71 & vv-miR171a & TGTTGGGACGGCTCAATCAAA & 5.67 & $\bullet$ \\
\hline Vvi-miR171b & TTGAGCCGCGTCAATATCTCC & 4.36 & vv-miR171b & TTGAGCCGCGTCAATATCTCC & 35.69 & $\uparrow$ \\
\hline Vvi-miR171c & GGATATTGGTGCGGTTCAATA & 5.15 & vv-miR171c & GGATATTGGTGCGGTTCAATA & 4.54 & $\bullet$ \\
\hline \multirow[t]{4}{*}{ Vvi-miR171d } & TTGATTGAGCCGTGCCAATAT & 5.15 & vv-miR171d & TTGATTGAGCCGTGCCAATAT & 2.53 & $\downarrow$ \\
\hline & & 0.00 & vv-miR171e & TGATTGAGCCGCGCCAATATC & 0.09 & $\boldsymbol{\Delta}$ \\
\hline & & 0.00 & vv-miR171f & TTGAGCCGCGCCAATATCACT & 2.01 & $\boldsymbol{\Delta}$ \\
\hline & & 0.00 & vv-miR171h & TTGAGCCGCGCCAATATCCCG & 0.96 & $\boldsymbol{\Delta}$ \\
\hline Vvi-miR171i & TGATTGAGCCGTGCCAATATC & 4.71 & vv-miR171i & TGATTGAGCCGTGCCAATATC & 85.43 & $\uparrow$ \\
\hline Vvi-miR172c & GGAGCATCATCAAGATTCACA & 0.09 & vv-miR172c & GGAGCATCATCAAGATTCACA & 103.40 & $\uparrow$ \\
\hline Vvi-miR172d & AGAATCTTGATGATGCTGCAT & 184.47 & vv-miR172d & AGAATCTTGATGATGCTGCAT & 117.63 & $\downarrow$ \\
\hline Vvi-miR319b & TTGGACTGAAGGGAGCTCCC & 0.35 & & & 0.00 & $\boldsymbol{\nabla}$ \\
\hline Vvi-miR319c & ATTGAATGATGCGGGAGACAA & 0.35 & vv-miR319c & ATTGAATGATGCGGGAGACAA & 20.68 & $\uparrow$ \\
\hline Vvi-miR319e & TाTGGACTGAAGGGAGCTCCT & 18.59 & vv-miR319e & TाTGGACTGAAGGGAGCTCCT & 7.94 & $\downarrow$ \\
\hline Vvi-miR319f & TGCTTGGACTGAAGGGAGC & 0.35 & vv-miR319f & TGCTTGGACTGAAGGGAGC & 3.40 & $\uparrow$ \\
\hline Vvi-miR319g & TTGGACTGAAGGGAGCTCCC & 0.26 & vv-miR319g & TTGGACTGAAGGGAGCTCCC & 1.40 & $\uparrow$ \\
\hline \multirow[t]{3}{*}{ Vvi-miR390 } & AAGCTCAGGAGGGATAGCGCC & 1.75 & vv-miR390 & AAGCTCAGGAGGGATAGCGCC & 160.73 & $\uparrow$ \\
\hline & & 0.00 & vv-miR393a & ATCATGCTATCCCTTAGGAAC & 1.66 & $\boldsymbol{\Delta}$ \\
\hline & & 0.00 & vv-miR393b & GGAGGAGGCATCCAAAGGGAT & 0.79 & $\boldsymbol{\Delta}$ \\
\hline Vvi-miR394a & TTGGCATTCTGTCCACCTCC & 2.71 & & & 0.00 & $\boldsymbol{\nabla}$ \\
\hline Vvi-miR394b & TATTGGCATTCTGTCCACCTCC & 2.53 & vv-miR394b & TATTGGCATTCTGTCCACCTCC & 0.09 & $\downarrow$ \\
\hline Vvi-miR394c & TTGGCATTCTGTCCACCTCC & 2.71 & vv-miR394c & TTGGCATTCTGTCCACCTCC & 0.79 & $\downarrow$ \\
\hline Vvi-miR395a & CTGAAGTGTTTGGGGGAACTC & 22.08 & & & 0.00 & $\boldsymbol{\nabla}$ \\
\hline Vvi-miR395b & CTGAAGTGTTTGGGGGAACTC & 22.08 & & & 0.00 & $\boldsymbol{\nabla}$ \\
\hline Vvi-miR395c & CTGAAGTGTTTGGGGGAACTC & 22.08 & & & 0.00 & $\boldsymbol{\nabla}$ \\
\hline Vvi-miR395d & CTGAAGTGTTTGGGGGAACTC & 22.08 & & & 0.00 & $\boldsymbol{\nabla}$ \\
\hline Vvi-miR395e & CTGAAGTGTTTGGGGGAACTC & 22.08 & & & 0.00 & $\boldsymbol{\nabla}$ \\
\hline Vvi-miR395f & CACTGAAGTGTTTGGGGGAAC & 22.08 & vv-miR395f & CACTGAAGTGTTGGGGGAAC & 0.09 & $\downarrow$ \\
\hline Vvi-miR395g & GTTCCCCTGAGCACTTCATTG & 22.16 & vv-miR395g & GTTCCCCTGAGCACTTCATTG & 0.52 & $\downarrow$ \\
\hline Vvi-miR395h & CTGAAGTGTTTGGGGGAACTC & 22.08 & & & 0.00 & $\boldsymbol{\nabla}$ \\
\hline
\end{tabular}


Table 2 Conserved Vv-miRNAs identified and normalized counts (NC) in control and GA $\mathrm{A}_{3}$ treated grapevine berries (Continued)

\begin{tabular}{|c|c|c|c|c|c|c|}
\hline Vvi-miR395i & CTGAAGTGTTTGGGGGAACTC & 22.08 & & & 0.00 & $\nabla$ \\
\hline Vvi-miR395j & CTGAAGTGTTTGGGGGAACTC & 22.08 & & & 0.00 & $\nabla$ \\
\hline Vvi-miR395k & GTTCCCTTGACCACTTCACTG & 22.08 & vv-miR395k & GTTCCCTTGACCACTTCACTG & 0.44 & $\downarrow$ \\
\hline Vvi-miR395I & CCCCTAGAGTTCCCCTGACCA & 22.08 & vv-miR395| & CCCCTAGAGTTCCCCTGACCA & 0.09 & $\downarrow$ \\
\hline Vvi-miR395m & CTGAAGTGTTTGGGGGAACTC & 22.08 & vv-miR395m & CTGAAGTGTTTGGGGGAACTC & 11.61 & $\downarrow$ \\
\hline Vvi-miR396a & CTCAAGAAAGCTGTGGGAGG & 25.04 & vv-miR396a & CTCAAGAAAGCTGTGGGAGG & 35.17 & $\uparrow$ \\
\hline Vvi-miR396b & TTCCACAGCTाCTTGAACTा & 53.23 & vv-miR396b & TTCCACAGCTTCTTGAACTT & 35.86 & $\downarrow$ \\
\hline Vvi-miR396c & TTCCACAGCTICTTGAACTG & 13.53 & vv-miR396c & TTCCACAGCTTCTTGAACTG & 18.41 & $\uparrow$ \\
\hline Vvi-miR396d & GTTCAATAAAGCTGTGGGAAG & 14.22 & vv-miR396d & GTTCAATAAAGCTGTGGGAAG & 6.20 & $\downarrow$ \\
\hline Vvi-miR397a & TCATTGAGTGCAGCGTTGATG & 7.59 & vv-miR397a & TCATTGAGTGCAGCGTTGATG & 29.41 & $\uparrow$ \\
\hline Vvi-miR398a & CAAGGGAGTGGCACCTGAGAACA & 0.09 & vv-miR398a & CAAGGGAGTGGCACCTGAGAACA & 6.98 & $\uparrow$ \\
\hline Vvi-miR398b & GGTGTGACCTGAGAATCACATG & 0.61 & vv-miR398b & GGTGTGACCTGAGAATCACATG & 0.26 & $\downarrow$ \\
\hline Vvi-miR398c & TGTGTTCTCAGGTCGCCCCTG & 0.61 & vv-miR398c & TGTGTTCTCAGGTCGCCCCTG & 1.13 & $\uparrow$ \\
\hline Vvi-miR399a & GTGTGATTCTCCTITGGCAGA & 0.70 & vv-miR399a & GTGTGATTCTCCITTGGCAGA & 1.57 & $\uparrow$ \\
\hline Vvi-miR399b & TGCCAAAGGAGAGTTGCCCTG & 0.26 & & & 0.00 & $\nabla$ \\
\hline Vvi-miR399c & TGCCAAAGGAGAGTTGCCCTG & 0.26 & vv-miR399c & TGCCAAAGGAGAGTTGCCCTG & 0.09 & $\downarrow$ \\
\hline Vvi-miR399d & TCTGCCAAAGGAGATITGCTC & 1.48 & vv-miR399d & TCTGCCAAAGGAGATTGCTC & 0.26 & $\downarrow$ \\
\hline Vvi-miR399e & TGCCAAAGGAGATTTGCCCGG & 3.58 & vv-miR399e & TGCCAAAGGAGATTTGCCCGG & 10.56 & $\uparrow$ \\
\hline Vvi-miR399g & TGCCAAAGGAGATTTGCCCCT & 9.08 & vv-miR399g & TGCCAAAGGAGATTTGCCCCT & 1.31 & $\downarrow$ \\
\hline Vvi-miR399h & TGCCAAAGGAGAATTGCCCTG & 0.52 & vv-miR399h & TGCCAAAGGAGAATTGCCCTG & 0.96 & $\uparrow$ \\
\hline Vvi-miR399i & CGCCAAAGGAGAGTTGCCCTG & 29.49 & vv-miR399i & CGCCAAAGGAGAGTTGCCCTG & 14.05 & $\downarrow$ \\
\hline Vvi-miR403a & TTAGATTCACGCACAAACTCG & 52.27 & & & 0.00 & $\nabla$ \\
\hline Vvi-miR403b & TTAGATTCACGCACAAACTCG & 52.62 & & & 0.00 & $\nabla$ \\
\hline Vvi-miR403c & CGCACAAACTCGTGATCTGTC & 52.27 & vv-miR403c & CGCACAAACTCGTGATCTGTC & 0.09 & $\downarrow$ \\
\hline Vvi-miR403d & AGTTTGTGCGCGAATCCAACC & 52.62 & vv-miR403d & AGTITGGCGCGAATCCAACC & 4.36 & $\downarrow$ \\
\hline Vvi-miR403e & TTAGATTCACGCACAAACTCGC & 52.18 & vv-miR403e & TTAGATTCACGCACAAACTCGC & 0.17 & $\downarrow$ \\
\hline Vvi-miR403f & TTAGATTCACGCACAAACTCG & 52.36 & vv-miR403f & TTAGATTCACGCACAAACTCG & 586.04 & $\uparrow$ \\
\hline Vvi-miR408 & ACGGGGACGAGGTAGTGCATG & 22.34 & vv-miR408 & ACGGGGACGAGGTAGTGCATG & 62.04 & $\uparrow$ \\
\hline Vvi-miR477 & TCCCTCAAAGGCTTCCAATTT & 98.25 & vv-miR477 & TCCCTCAAAGGCTTCCAATTT & 21.82 & $\downarrow$ \\
\hline Vvi-miR479 & TGTGGTATTGGTTCGGCTCATC & 2922.60 & vv-miR479 & TGTGGTATTGGTTCGGCTCATC & 1131.41 & $\downarrow$ \\
\hline Vvi-miR482 & AATTGGAGAGTAGGAAAGCTT & 45.11 & vv-miR482 & AATTGGAGAGTAGGAAAGCTT & 1056.46 & $\uparrow$ \\
\hline Vvi-miR535a & TGACAACGAGAGAGAGCACGC & 763.18 & & & 0.00 & $\nabla$ \\
\hline Vvi-miR535b & ACGAGAGAGAGCACGCTAGTCAG & 763.18 & vv-miR535b & ACGAGAGAGAGCACGCTAGTCAG & 0.09 & $\downarrow$ \\
\hline Vvi-miR535c & TGACAACGAGAGAGAGCACGC & 763.18 & vv-miR535c & TGACAACGAGAGAGAGCACGC & 83.42 & $\downarrow$ \\
\hline Vvi-miR828a & AGATGCTCATTTGAGGAAGCAA & 1.13 & vv-miR828a & AGATGCTCATTGAGGAAGCAA & 5.32 & $\uparrow$ \\
\hline
\end{tabular}

Notes: $\uparrow, \downarrow, \boldsymbol{\Lambda}, \boldsymbol{\nabla}, \bullet$ denote up-regulated, down-regulated, induced, repressed, un-affected, respectively.

by Vvi-miR156, Vvi-miR168, Vvi-miR167, Vvi-miR479, Vvi-miR482 families, whose redundancies were more than several ten thousands. However, other miRNA families like Vvi-miR393, Vvi-miR394, Vvi-miR398 and VvimiR399 had only a few or tens of reads. Interestingly, the number and abundance of members of different VvimiRNA families between control and $\mathrm{GA}_{3}$ libraries were significant divergence (Table 2; Figure 3), which in turn could reflect the discrepancy in their potential functions during the development of grapevine berries responsive to gibberellin.

Bioinformatics analysis of the sequencing data could identify novel Vvi-miRNAs based on the criteria of novel miRNA annotations developed by Meyers et al. (2008) [38]. The genomic sequences with flanking un-announced sequences were extracted. Among these sequences, 90 candidate Vvi-miRNAs were firstly uncovered, and the hairpin structures of their precursors' could be predicted 


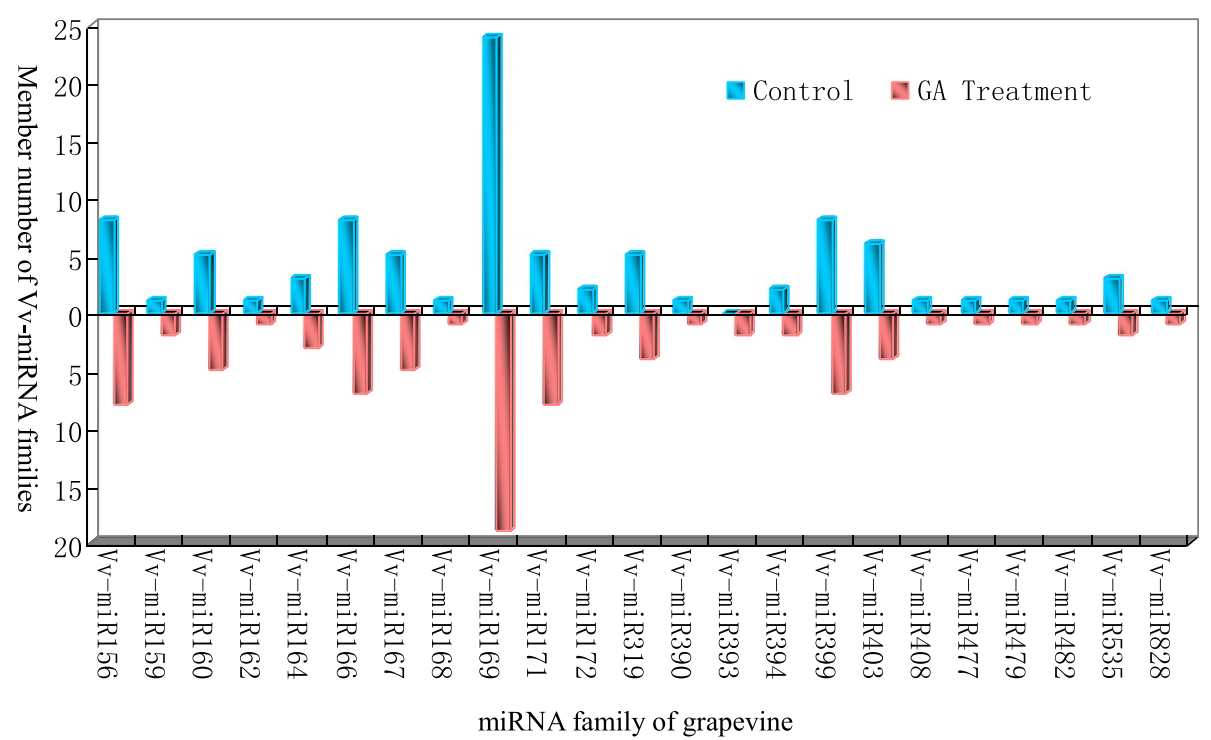

Figure 3 Number of members from Vv-miRNA families in control and $\mathrm{GA}_{3}$ treated grapevine berries.

(Additional file 1). The miRNA* sequences available is of vital evidence supporting the release of miRNA duplex from the predicted foldback structure [16]. Among these 90 potential novel Vvi-miRNAs, 28 had their Vvi-miRNA* sequences were detected (Additional file 2), and the sequences of 45 novel potential Vvi-miRNAs started with a 5' uridine, the important feature of miRNAs (Additional file 2). All these confirmed the existence of these novel candidate VvimiRNAs in grapevine. In this study, it was also noted that the novel Vvi-miRNAs had fewer reads than the conserved ones. $72 \%$ of the former had only several or dozens of copies except for Vvi-miRC20, Vvi-miRC36, Vvi-miRC43, Vvi-miRC44, Vvi-miRC45, Vvi-miRC46, Vvi-miRC50, VvimiRC51, Vvi-miRC52, Vvi-miRC71, and Vvi-miRC74 that were sequenced more than one thousand times (Additional file 2). This agreed with other previously reported results $[10,11,36]$, where most novel species-specific miRNAs were usually expressed at lower levels than their conserved counterparts and were much more spatiotemporally expressed.

\section{Discovery of miRNAs responsive to exogenous $\mathrm{GA}_{3}$ in grapevine}

To identify Vvi-miRNAs responsive to exogenous $\mathrm{GA}_{3}$, we normalized the counts of reads sequenced based on the systematical analysis, and compared the members and the normalized counts (NC) of different members of Vvi-miRNA families between the control and $\mathrm{GA}_{3}$ libraries. The results revealed that seven known Vvi-miRNAs (Vvi-miR159b, Vvi-miR160f, Vvi-miR171e, Vvi-miR171f, Vvi-miR171h, VvimiR393a and Vvi-miR393b) were found only in the $\mathrm{GA}_{3}$ treated grapevine tissues not in the control, suggesting they were probably those induced by $\mathrm{GA}_{3}$; another 21 known Vvi-miRNAs (Vvi-miR160d, Vvi-miR166c, Vvi-miR169a,
Vvi-miR169e, Vvi-miR169j, Vvi-miR169m, Vvi-miR169s, Vvi-miR319b, Vvi-miR394a, Vvi-miR395a, Vvi-miR395b, Vvi-miR395c, Vvi-miR395d, Vvi-miR395e, Vvi-miR395h, Vvi-miR395i, Vvi-miR395j, Vvi-miR399b, Vvi-miR403a, VvimiR403b and Vvi-miR535a) were strongly repressed in $\mathrm{GA}_{3}$ treatment for they could only be detected in the control but not in the $\mathrm{GA}_{3}$ treatment (Table 2).

Thirty novel Vvi-miRNAs (Vvi-miRC02, Vvi-miRC03, Vvi-miRC05, Vvi-miRC08, Vvi-miRC10, Vvi-miRC11, Vvi-miRC13, Vvi-miRC14, Vvi-miRC16, Vvi-miRC17, Vvi-miRC21-Vvi-miRC26, Vvi-miRC33, Vvi-miRC39, Vvi-miRC41, Vvi-miRC42, Vvi-miRC54, Vvi-miRC55, Vvi-miRC57, Vvi-miRC59, Vvi-miRC61, Vvi-miRC64, Vvi-miRC65, Vvi-miRC68 and Vvi-miRC72) were found to be responsive to exogenous $\mathrm{GA}_{3}$, for they could only be detected in $\mathrm{GA}_{3}$ treated grapevines (Table 2); while another 16 novel Vvi-miRNAs (from Vvi-miRC75 to VvimiRC90) were repressed by exogenous GA3, and found only in control but not in $\mathrm{GA}_{3}$ treatment (Table 2). These results demonstrate that these groups of Vvi-miRNAs could be responsive to $\mathrm{GA}_{3}$ treatment.

Further comparison of the normalized counts of VvimiRNAs identified in $\mathrm{GA}_{3}$ treated and the control plants indicated that many Vvi-miRNAs exhibited drastic variations (increases/decreases over several times) in expression frequencies. As shown in Table 2 and Table 3, the expression of 137 Vvi-miRNAs was strongly responsive to exogenous $\mathrm{GA}_{3}$, with 58 of them induced by $\mathrm{GA}_{3}$, showing conspicuously up-regulated expression under $\mathrm{GA}_{3}$ treatment (pointed as $\uparrow$ in Table 2 and 3).Conversely, 51 Vvi-miRNAs were repressed by exogenous $\mathrm{GA}_{3}$ (pointed as $\downarrow$ in Table 2 and 3 ), leading to drastic reduction in their expression 
Table 3 Comparison of normalized counts (NC) of novel Vv-miRNAs between $\mathrm{GA}_{3}$ treated and control grapevine berries

\begin{tabular}{|c|c|c|c|c|}
\hline \multirow[t]{2}{*}{ miRNA ID } & \multirow[t]{2}{*}{ Mature sequences } & \multicolumn{2}{|c|}{ NC } & \multirow{2}{*}{$\begin{array}{l}\text { Identity of } \\
\text { frequency }\end{array}$} \\
\hline & & Control & GA treatment & \\
\hline Vvi-miRC01 & CTATGTTATAGGATCTTGGAT & 9.16 & 17.19 & $\uparrow$ \\
\hline Vvi-miRC01* & CCAAGATACTATAACATGGTC & 0.00 & 0.17 & $\boldsymbol{\Delta}$ \\
\hline Vvi-miRC02 & TCCCTTTGGAAGTGCTAAGCG & 0.00 & 1.83 & $\boldsymbol{\Delta}$ \\
\hline Vvi-miRC03 & AGTGGTGGCAAGGATGAGCAA & 0.00 & 0.52 & $\boldsymbol{\Delta}$ \\
\hline Vvi-miRC04 & TाGGAATGATTTGTTGATGA & 4.62 & 1.48 & $\downarrow$ \\
\hline Vvi-miRC05 & AAGATCTCCCATTGCATCTGA & 0.00 & 0.52 & $\boldsymbol{\Delta}$ \\
\hline Vvi-miRC06 & TITITGGTTATGGTTGGCTG & 0.61 & 1.40 & $\uparrow$ \\
\hline Vvi-miRC07 & CTCAAGAAAGCTGTGGGAAAA & 0.61 & 1.05 & $\uparrow$ \\
\hline Vvi-miRC07* & TITCCACATCTTTCTTGAACT & 0.09 & 0.17 & $\bullet$ \\
\hline Vvi-miRC08 & AGAAGAACAAGTAGACTGAGC & 0.00 & 0.96 & $\boldsymbol{\Delta}$ \\
\hline Vvi-miRC09 & TTATATAGGCTTTGAGGATGGA & 0.00 & 1.92 & $\Delta$ \\
\hline Vvi-miRC10 & TITAAAAAGGTTCGTCATTC & 0.00 & 0.87 & $\Delta$ \\
\hline Vvi-miRC11 & CCGTGACAAGTGGTATCAGAG & 0.00 & 1.13 & $\boldsymbol{\Delta}$ \\
\hline Vvi-miRC12 & TCTGAAGTTTGAAGAGCTGTG & 4.97 & 10.56 & $\uparrow$ \\
\hline Vvi-miRC12* & AGAGCAATCTACGAACAACAGGAA & 0.09 & 0.09 & $\bullet$ \\
\hline Vvi-miRC13 & TTGGCTTGGAGATGGATCATT & 0.00 & 10.56 & $\boldsymbol{\Delta}$ \\
\hline Vvi-miRC14 & TTGGCTTGGAGATGGATCATT & 0.00 & 10.56 & $\boldsymbol{\Delta}$ \\
\hline Vvi-miRC15 & TCAATTGAGAGCTGGAAGAA & 0.61 & 0.70 & $\bullet$ \\
\hline Vvi-miRC16 & ATATTGGTAAATGAATGTTCG & 0.00 & 1.40 & $\boldsymbol{\Delta}$ \\
\hline Vvi-miRC17 & AATTTCTTATGTTCATGATTG & 0.00 & 0.79 & $\Delta$ \\
\hline Vvi-miRC18 & AAGAGCAGTTGAACTGAAGCA & 0.70 & 1.57 & $\uparrow$ \\
\hline Vvi-miRC19 & TCTGTCGCAGGAGAGATGATGC & 1.66 & 4.19 & $\uparrow$ \\
\hline Vvi-miRC20 & GGAATGGGCTGATTGGGATA & 2551.57 & 760.03 & $\downarrow$ \\
\hline Vvi-miRC20* & TTCCCAATGCCGCCCATTCCAA & 94.07 & 208.55 & $\uparrow$ \\
\hline Vvi-miRC21 & CCAAGAGGGTGGAGTTCAGAT & 0.00 & 1.48 & $\Delta$ \\
\hline Vvi-miRC21* & CTGAACTCTCTCCCTCATGGCC & 0.00 & 0.87 & $\Delta$ \\
\hline Vvi-miRC22 & CTAAATTGCTTCGGGTCCTGC & 0.00 & 6.63 & $\boldsymbol{\Delta}$ \\
\hline Vvi-miRC22* & AGGAGATGAGGTATGTTTACAT & 0.00 & 5.93 & $\boldsymbol{\Delta}$ \\
\hline Vvi-miRC23 & AAACATGAGTCTGGACCTTGA & 0.00 & 0.79 & $\boldsymbol{\Delta}$ \\
\hline Vvi-miRC24 & AAACATGAGTCTGGACCTTGA & 0.00 & 0.79 & $\boldsymbol{\Delta}$ \\
\hline Vvi-miRC25 & TCTGTITCACTCTCATTAAG & 0.00 & 1.13 & $\Delta$ \\
\hline Vvi-miRC25* & TAGTGAGAATGAGTTGGGGAAG & 0.00 & 0.09 & $\boldsymbol{\Delta}$ \\
\hline Vvi-miRC26 & TCGGAGAAGTGTGATGTGTAT & 0.00 & 0.70 & $\Delta$ \\
\hline Vvi-miRC27 & ATACCATGTGGAAAAGAGGAATC & 6.72 & 5.85 & $\bullet$ \\
\hline Vvi-miRC28 & ATTGGCAGAATATTCAAGGTTT & 0.44 & 0.87 & $\uparrow$ \\
\hline Vvi-miRC29 & TTATTAGGAGGACATTTAGGTAT & 2.88 & 3.49 & $\bullet$ \\
\hline Vvi-miRC30 & TGCGGGTGGAAGAGAAGGAAG & 5.67 & 3.49 & $\downarrow$ \\
\hline Vvi-miRC31 & TTCCTGCGGTTCTCGGCGAC & 0.96 & 0.96 & $\bullet$ \\
\hline Vvi-miRC32 & TITCCTATGATTTCTTGGCA & 0.87 & 0.79 & $\bullet$ \\
\hline Vvi-miRC32* & CTGGGAAAGCGTGGGAAAACA & 0.00 & 0.09 & $\Delta$ \\
\hline Vvi-miRC33 & TTCCTATCGTTCCCGGGATTT & 0.00 & 1.22 & $\boldsymbol{\Delta}$ \\
\hline Vvi-miRC34 & TGACCGGCTCTTATCTCTCATG & 1.48 & 3.93 & $\uparrow$ \\
\hline Vvi-miRC34* & TGAAGATAAAGAGTCTCGTCTGG & 0.35 & 0.09 & $\downarrow$ \\
\hline
\end{tabular}


Table 3 Comparison of normalized counts (NC) of novel Vv-miRNAs between $\mathrm{GA}_{3}$ treated and control grapevine berries (Continued)

\begin{tabular}{|c|c|c|c|}
\hline Vvi-miRC35 & GGAATGGATGGCATGGGAACCA & 0.52 & 0.52 \\
\hline Vvi-miRC36 & TGAGTAGTGGACTATCGCATG & 14.57 & 1728.01 \\
\hline Vvi-miRC36* & TGAGATAAGTCTGCTGCTCCAT & 0.61 & 94.76 \\
\hline Vvi-miRC37 & TGGATGCATGTAGCTTGTCAA & 16.06 & 0.87 \\
\hline Vvi-miRC37* & GACAAGTTACATACATCCAAG & 1.05 & 0.17 \\
\hline Vvi-miRC38 & TCCTTCGGCGTCGGCAAATCC & 1.75 & 1.22 \\
\hline Vvi-miRC39 & AAGGGTTCTCACAGAGTTTA & 0.00 & 0.79 \\
\hline Vvi-miRC39* & AGCTCTGTTGGACTCTCTTTG & 0.00 & 0.17 \\
\hline Vvi-miRC40 & GAGGAGAATGTAGTGGGGTTA & 0.52 & 0.44 \\
\hline Vvi-miRC41 & CTTTGATCAGATATTGGATTG & 0.00 & 1.40 \\
\hline Vvi-miRC41* & AGCAGAGTTTGATAGAGGGC & 0.00 & 0.09 \\
\hline Vvi-miRC42 & AATGACATGAGTTGGAACTAA & 0.00 & 0.87 \\
\hline Vvi-miRC43 & GTTGGAAGCCGGTGGGGGACC & 4389.70 & 425.65 \\
\hline Vvi-miRC44 & GTTGGAAGCCGGTGGGGGACC & 4389.70 & 425.65 \\
\hline Vvi-miRC45 & GTTGGAAGCCGGTGGGGGACC & 4389.70 & 425.65 \\
\hline Vvi-miRC46 & GTTGGAAGTCGGTGGGGGAAC & 1990.92 & 272.69 \\
\hline Vvi-miRC47 & GGCGATTGTAAATATGGGTAA & 3.75 & 1.13 \\
\hline Vvi-miRC48 & TCTAGATTTGGAAGTAGGTCA & 0.70 & 0.44 \\
\hline Vvi-miRC49 & GTTGGAAGTCGGTGGGGGACC & 1089.01 & 72.95 \\
\hline Vvi-miRC50 & GTTGGAAGCCGGTGGGGGACC & 4389.70 & 425.65 \\
\hline Vvi-miRC51 & TGGGCTTGTGGAGAAGAAAGTGA & 0.96 & 0.52 \\
\hline Vvi-miRC52 & CATGGGCGGTTTGGTAAGAGG & 2805.93 & 1401.92 \\
\hline Vvi-miRC52* & TCTTACCAACACСТСССАТTCC & 140.49 & 198.43 \\
\hline Vvi-miRC53 & GGTATGGGAGGATTGGGGAGA & 1305.32 & 437.43 \\
\hline Vvi-miRC53* & TTCCCAAGACCCCCCATGCCAA & 62.48 & 327.23 \\
\hline Vvi-miRC54 & TCATACCTCGATCTTCGGTTTC & 0.00 & 0.70 \\
\hline Vvi-miRC54* & AATCTGAGATCGAGAATGAAA & 0.00 & 0.09 \\
\hline Vvi-miRC55 & ATTCGAACTCAAGACTAAGGT & 0.00 & 41.54 \\
\hline Vvi-miRC56 & GAAGCTCTTGAGGGGGACTG & 378.18 & 60.38 \\
\hline Vvi-miRC56* & ACTCTCCCTCAAGGGCTTCTG & 12.13 & 1.31 \\
\hline Vvi-miRC57 & AGGTGTAGATGCAAGTGCAGA & 0.00 & 1.05 \\
\hline Vvi-miRC58 & TTTAATTTACTAGAGATCTCT & 1.13 & 1.40 \\
\hline Vvi-miRC59 & GGAGTGAAATTGCAGTGACGG & 0.00 & 1.13 \\
\hline Vvi-miRC60 & TCAGCAGGAATTGGACCAGAA & 2.88 & 3.75 \\
\hline Vvi-miRC61 & ACAGTAGGAAATTGAAAGAGA & 0.00 & 0.70 \\
\hline Vvi-miRC61* & TCTTCATTTTCCTACTTITT & 0.00 & 0.52 \\
\hline Vvi-miRC62 & AAAGGCGAAGAAAAAGAAGATA & 1.75 & 0.79 \\
\hline Vvi-miRC63 & AATATGGAGGACTGTGTTCTT & 0.61 & 1.75 \\
\hline Vvi-miRC63* & GAACTCAGTTCCGGTACCATCTTCA & 0.09 & 0.09 \\
\hline Vvi-miRC64 & TTGGATTCGCGCACAAACTCG & 0.00 & 1.13 \\
\hline Vvi-miRC65 & TTGGATTCGCGCACAAACTCG & 0.00 & 1.13 \\
\hline Vvi-miRC66 & CAGCAGTTGCTATTGTGGTTG & 0.87 & 8.38 \\
\hline Vvi-miRC67 & AGAAGAGAGAGAGTACAGCTA & 1.31 & 9.60 \\
\hline Vvi-miRC68 & TGGTACCAGGAGGGCAACTGTC & 0.00 & 1.05 \\
\hline
\end{tabular}


Table 3 Comparison of normalized counts (NC) of novel Vv-miRNAs between $\mathrm{GA}_{3}$ treated and control grapevine berries (Continued)

\begin{tabular}{|c|c|c|c|c|}
\hline Vvi-miRC68* & TGTTGCCCTCCTGGTACCATC & 0.00 & 0.09 & $\boldsymbol{\Delta}$ \\
\hline Vvi-miRC69 & TCAAGGGTCGAACGGCTITGC & 1.31 & 2.36 & $\uparrow$ \\
\hline Vvi-miRC70 & TTATGTGAGTGTTCGGCAAATC & 0.79 & 2.62 & $\uparrow$ \\
\hline Vvi-miRC71 & TTAGATGATCATCAACAAACA & 436.74 & 517.54 & $\bullet$ \\
\hline Vvi-miRC71* & TITTGTTGCTGGTCATCTAGTC & 2.09 & 3.05 & $\bullet$ \\
\hline Vvi-miRC72 & TGCTTATTAGGTCTGCTGGCA & 0.00 & 0.61 & $\boldsymbol{\Delta}$ \\
\hline Vvi-miRC73 & TCAAAAGAGAAAATGTGGATG & 0.52 & 0.79 & $\bullet$ \\
\hline Vvi-miRC73* & ТССАТСТTСТСТСПIITACA & 0.00 & 0.09 & $\boldsymbol{\Delta}$ \\
\hline Vvi-miRC74 & TCGCAGGAGAGATGACGCCGT & 52.97 & 110.21 & $\uparrow$ \\
\hline Vvi-miRC74* & AGCATCATTTCTCCTGCATAG & 1.13 & 4.28 & $\uparrow$ \\
\hline Vvi-miRC75 & ATATTAGCAGCTGAGAACACA & 1.40 & 0.00 & $\boldsymbol{\nabla}$ \\
\hline Vvi-miRC76 & CAGGACTGGCAGTGATGGTTA & 1.13 & 0.00 & $\nabla$ \\
\hline Vvi-miRC77 & GTGTITGCAGGATCAGACGG & 0.70 & 0.00 & $\boldsymbol{\nabla}$ \\
\hline Vvi-miRC78 & TGGCTGAGAACTTGATGGTTA & 2.71 & 0.00 & $\boldsymbol{\nabla}$ \\
\hline Vvi-miRC79 & TTCAAGTCAAAGTCGAACAAG & 0.87 & 0.00 & $\nabla$ \\
\hline Vvi-miRC80 & AGCGAAGTAGTTGTAGGGCTT & 0.96 & 0.00 & $\boldsymbol{\nabla}$ \\
\hline Vvi-miRC81 & TTCGGAGGGAACTGACCGGTT & 0.70 & 0.00 & $\boldsymbol{\nabla}$ \\
\hline Vvi-miRC82 & TGCCAAGAAGCACATTCCTCC & 16.84 & 0.00 & $\boldsymbol{\nabla}$ \\
\hline Vvi-miRC $82^{*}$ & AGGAATGTGCTTCTTGGCATA & 0.09 & 0.00 & $\nabla$ \\
\hline Vvi-miRC83 & CAAGTGTGGGATTTTGGGTGGCT & 0.52 & 0.00 & $\boldsymbol{\nabla}$ \\
\hline Vvi-miRC84 & GCAGCATCATGAAGATTCACA & 0.52 & 0.00 & $\nabla$ \\
\hline Vvi-miRC84* & GGAATCTTGATGATGCTGCAT & 0.17 & 0.00 & $\boldsymbol{\nabla}$ \\
\hline Vvi-miRC85 & AGGTGCAGGTGAAGGTGCAGA & 1.75 & 0.00 & $\nabla$ \\
\hline Vvi-miRC85* & TGCATTGCACCTGCACCTA & 0.96 & 0.00 & $\boldsymbol{\nabla}$ \\
\hline Vvi-miRC86 & GTAGCATCATCAAGATTCACA & 1.66 & 0.00 & $\nabla$ \\
\hline Vvi-miRC87 & GGAATGTTGTCTGGCTCGAGGT & 0.70 & 0.00 & $\boldsymbol{\nabla}$ \\
\hline Vvi-miRC88 & ATGTATTTGAGGGAAAGCAAA & 0.44 & 0.00 & $\nabla$ \\
\hline Vvi-miRC88* & TGTITCCCTCAAAAACATGT & 0.09 & 0.00 & $\boldsymbol{\nabla}$ \\
\hline Vvi-miRC89 & CTGCGGGTGGAAAAGGATTAGGC & 6.81 & 0.00 & $\boldsymbol{\nabla}$ \\
\hline Vvi-miRC89* & СTCATCCTITCCATCGGCAGCA & 0.35 & 0.00 & $\nabla$ \\
\hline Vvi-miRC90 & TCTCAGCAACCAAGTAGAGCC & 5.93 & 0.00 & $\boldsymbol{\nabla}$ \\
\hline
\end{tabular}

Notes: $\uparrow, \downarrow, \boldsymbol{\Delta}, \boldsymbol{\nabla}, \bullet$ denote up-regulated, down-regulated, induced, repressed, un-affected, respectively; the symbol * denotes the complementary strands of miRNAs.

levels in $\mathrm{GA}_{3}$-treated grapevines. An interesting revelation was that diverse members of the same VvimiRNA family could exhibit conspicuous discrepancy in their responses to exogenous $\mathrm{GA}_{3}$. This aspect is best exemplified by Vvi-miR166 family, where Vvi$\mathrm{miR} 166 \mathrm{~d} / \mathrm{e} / \mathrm{f} / \mathrm{g}$ had more than 246,000 reads in control samples, but possessed only several dozen or even a little more reads in the $\mathrm{GA}_{3}$ treatment samples, while Vvi-miR166b was detected 37,952 times in control and 176,156 times in $\mathrm{GA}_{3}$ treatment (Table 2). VvimiR166h, however, had no distinct variations between the control and $\mathrm{GA}_{3}$ treatment (Table 2). Similar situations were found in other Vvi-miRNA families like Vvi-
miR156, Vvi-miR164, Vvi-miR167, Vvi-miR403 and Vvi-miR535 (Table 2), suggesting the Vvi-miRNAs responsive to exogenous $\mathrm{GA}_{3}$ application possess multiple aspects and functions during the development of grapevine berries.

\section{Expression patterns of Vvi-miRNAs responsive to} exogenous $\mathrm{GA}_{3}$ during grapevine berry development Spatiotemporal expression of grapevine miRNAs could not only provide clues to their physiological functions, but also give fundamental evidence supporting the existence of the miRNAs in grapevine. In this study, 53 Vvi-miRNAs (27 conserved Vvi-miRNAs and 26 novel candidate Vvi- 
miRNAs) detected in grapevine berries treated with $\mathrm{GA}_{3}$ were subjected to qRT-PCR expression analysis as described in Section 2. This could also be applied to the analysis of the degree of response of these Vvi-miRNAs to $\mathrm{GA}_{3}$ treatments. We screened for the expression profiles of miRNAs responsive to $\mathrm{GA}_{3}$ treatments in the diverse stages of grapevine berries by qRT-PCR. The results showed that the expression levels of 15 Vvi-miRNAs (Vvi-miR169d, Vvi-miR319c, Vvi-miR393a, Vvi-miR396a, Vvi-miR398a, Vvi-miR399a, Vvi-miRC03, Vvi-miRC04, Vvi-miRC05, VvimiRC08, Vvi-miRC10, Vvi-miRC13, Vvi-miRC19, VvimiRC26 and Vvi-miRC37) were up-regulated by $\mathrm{GA}_{3}$, while 13 (Vvi-miR167a, Vvi-miR171d, Vvi-miR395f, Vvi-miR397a, Vvi-miR408, Vvi-miR482, Vvi-miRC06, Vvi-miRC14, Vvi-miRC15, Vvi-miRC24, Vvi-miRC27, VvimiRC30, Vvi-miRC38) were down-regulated by $\mathrm{GA}_{3}$, and seven (Vvi-miR156d, Vvi-miR166h, Vvi-miR390, VvimiR477, Vvi-miRC23, Vvi-miRC29, and Vvi-miRC36) were un-affected by $\mathrm{GA}_{3}$ (see Figure 4).

Further analysis of the expression results of 16 known Vvi-miRNAs responsive to $\mathrm{GA}_{3}$ both from qRT-PCR and high throughput sequencing revealed that the qRT-PCR expressions of 11 were consistent with the results from high throughput sequencing, which could not only demonstrated the reliability of these two technologies, but confirm the characteristics of these known $\mathrm{GA}_{3}$ responsive Vvi-miRNAs. On the other hand, it was discernible that the expression analysis results of 18 novel $\mathrm{GA}_{3}$ responsive Vvi-miRNAs from both qRT-PCR and high throughput sequencing were not of the same phenomenon, for only three (Vvi-miRC19, Vvi-miRC14 and Vvi-miRC29) was in the same way. This discrepancy in behavior of conserved and novel Vvi-miRNAs requires further research; while the consistency of the expression results, for the most conserved and a few novel Vvi-miRNAs, from the assays of the qRT-PCR and high throughput may provide some evidence supporting the fact about these Vvi-miRNAs responsive to $\mathrm{GA}_{3}$.

\section{Characterization of potential target genes for novel Vvi-miRNAs responsive to $\mathrm{GA}_{3}$}

To further comprehend the functions of these newly identified species-specific Vvi-miRNAs, it was essential to search for their target genes. Following the rules suggested by Schwab et al. (2005) [39], we searched the grapevine transcript database (http://www.genoscope.cns.fr/spip), and predicted a total of 117 putative target genes for 29 novel Vvi-miRNAs (Additional file 3). Among the 29 novel VvimiRNAs, 11 had multiple target genes, as exemplified by Vvi-miRC03 with 19 target genes, indicating these VvimiRNAs might possess comprehensive functions in grapevine. Based on orthologous functional annotation in other plants, these target genes seemed to be functionally involved in glucose metabolism (DNA glycosylase domain- containing protein), aromatic substance biosynthesis in berry (lipoxygenase, nigralipoxygenase), signal transduction (receptor-like protein kinase, serine-threonine protein kinase, protein phosphatase), stress resistance (ankyrin repeat-containing protein, disease resistance protein,ccnbs-lrrresistance protein, domain-containing disease resistance protein, leucine-rich repeat receptor-like protein kinase), etc. (Figure 5; Additional file 3). We also noted that there were a large number of potential target genes with unknown functions for novel Vvi-miRNAs, especially those responsive to $\mathrm{GA}_{3}$ (Additional file 3). More in depth investigation on their functions would be essential for thorough understanding of the mechanisms of grapevine flower and fruit development and of the formation of berry quality.

\section{Verification of potential Vvi-miRNA target genes using 5'-RLM-RACE}

To verify the nature of potential miRNA targets and to study the Vvi-miRNAs' regulation on their target genes, a modified RLM-RACE experiment was set up and used to map the cleavage sites in four predicted Vvi-miRNA target genes. Results showed that the cleavage sites of these four miRNA target genes GSVIVT01000639001, GSVIVT01026728001, GSVIVT01037667001 and GSVI VT01037667001 for Vvi-miRC15, Vvi-miRC23, Vvi-miRC60 and Vvi-miRC72 in this study is at the nucleotide that pairs with the $9^{\text {th }}$ and/or $10^{\text {th }}$ and/or $11^{\text {th }}$ nucleotide of the corresponding miRNAs (Figure 6), consistent with previous related reports $[7,16,18,19,40]$. The GSVIVT01000639001, GSVIVT01026728001, GSVIVT01037667001 and GSVIV T01037667001 were confirmed as the true targets of Vvi-miR015, Vvi-miR023, Vvi-miR060 andVvi-miR072, respectively. Functional analysis indicated that GSVIV T01000639001, GSVIVT01026728001, GSVIVT01037667 001 and GSVIVT01037667001 could be of cc-nbs-lrr resistance protein, nbs-lrrresistance protein, leucine-rich repeat family protein, and lipoxygenase (Additional file 3).

\section{Discussion}

To verify the hypothesis that miRNAs play some role inregulating plant response to $\mathrm{GA}_{3}$ [28], two small RNA libraries from grapevine berries treated with $\mathrm{GA}_{3}$ and without $\mathrm{GA}_{3}$ were constructed and used for high-throughput sequencing of sRNAs, from which a total of 212 Vvi-miRNAs (both known and novel), with 137 of them being firstly found to be responsive to $\mathrm{GA}_{3}$ in grapevine. Further comparison of our dataset from this work to Vvi-miRNAs reported earlier by us [20] revealed that since the grapevine materials used in these studies was the same grapevine cultivar, most of the Vvi-miRNAs could be observed in these two studies, while only a few members (such as Vvi-miR171f, Vvi-miR171h) could be discovered in one of both studies, which may be derived from the differences in the development stages of 

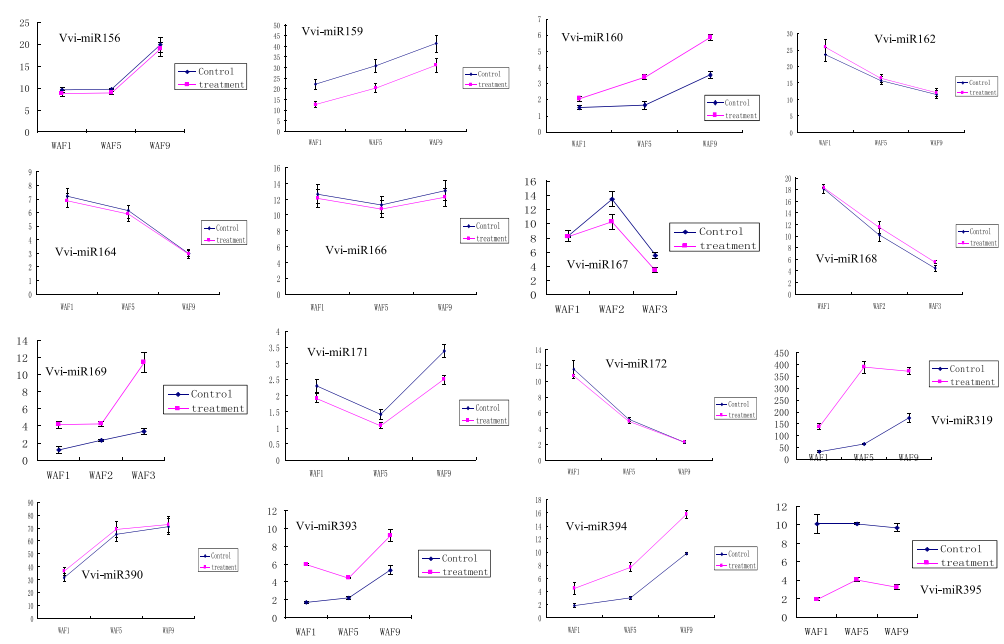

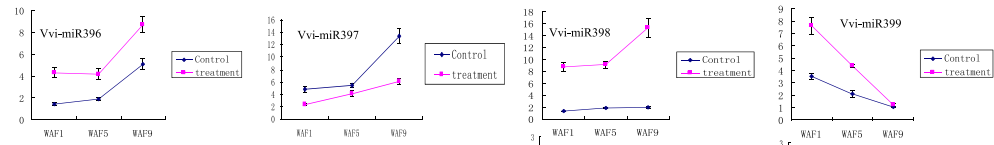
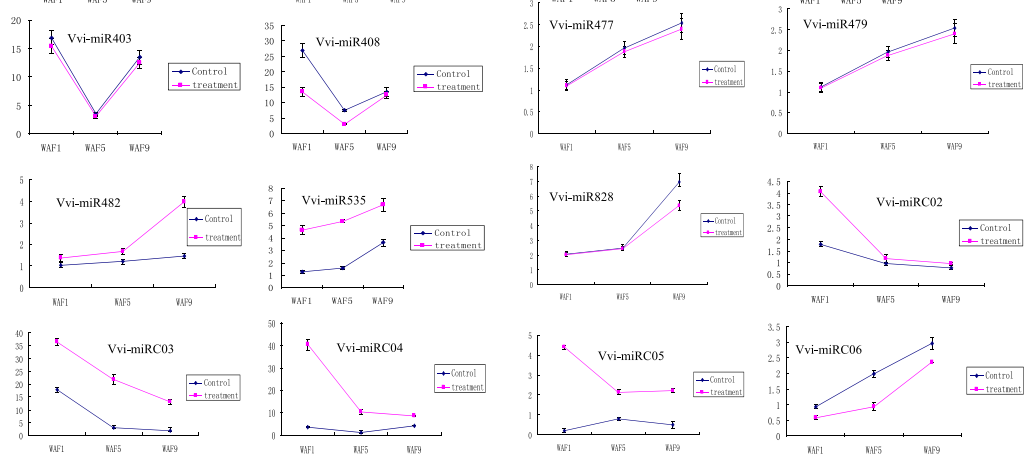

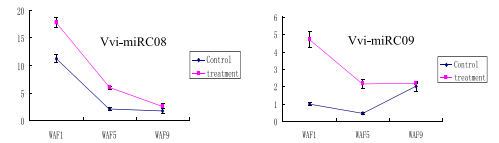

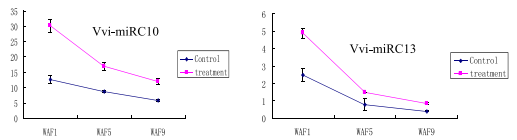

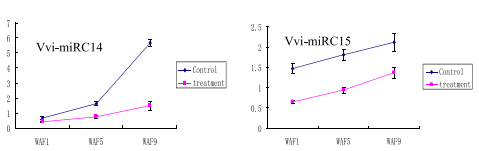

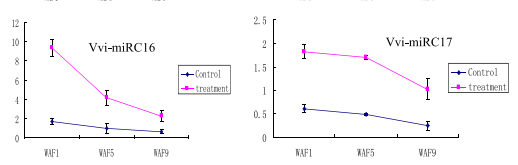

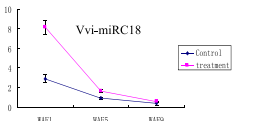

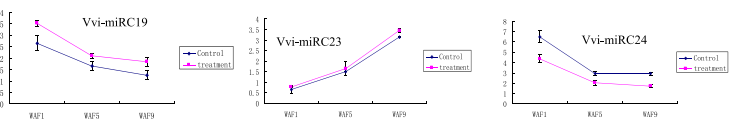

VIvi-miRC26

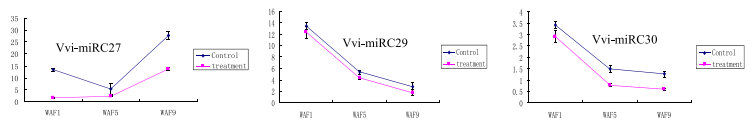

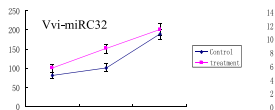

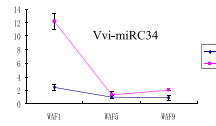

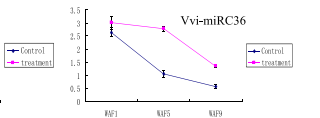
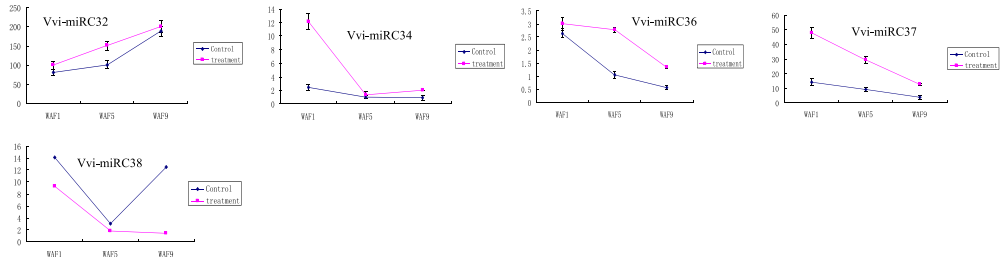

Figure 4 Expression patterns of miRNAs in grapevine berries underdifferent $\mathrm{GA}_{3}$ treatments and control. 


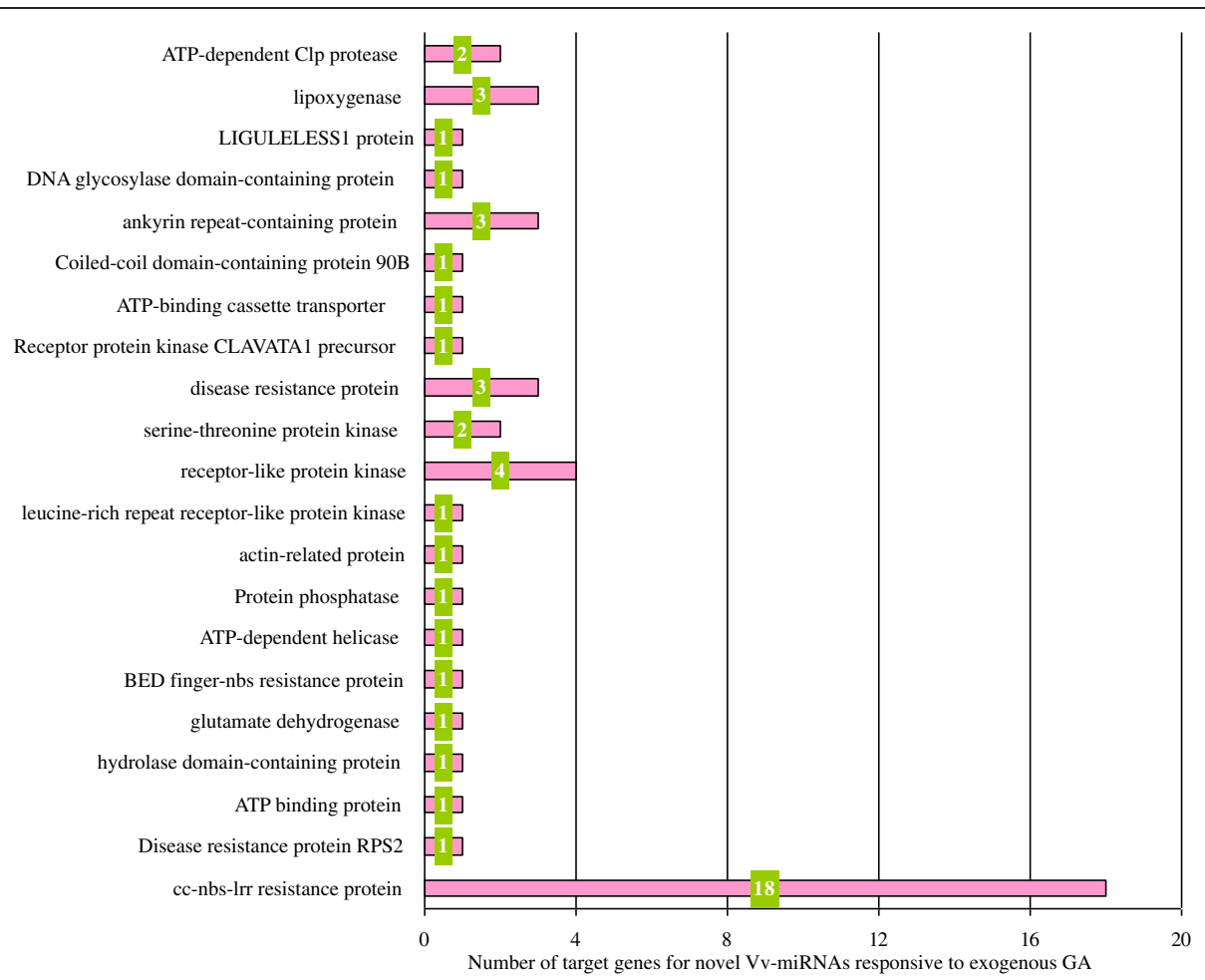

Figure 5 Functional classification of target genes for novel $\mathrm{Vv}$-miRNAs responsive to exogenous $\mathrm{GA}_{3}$.

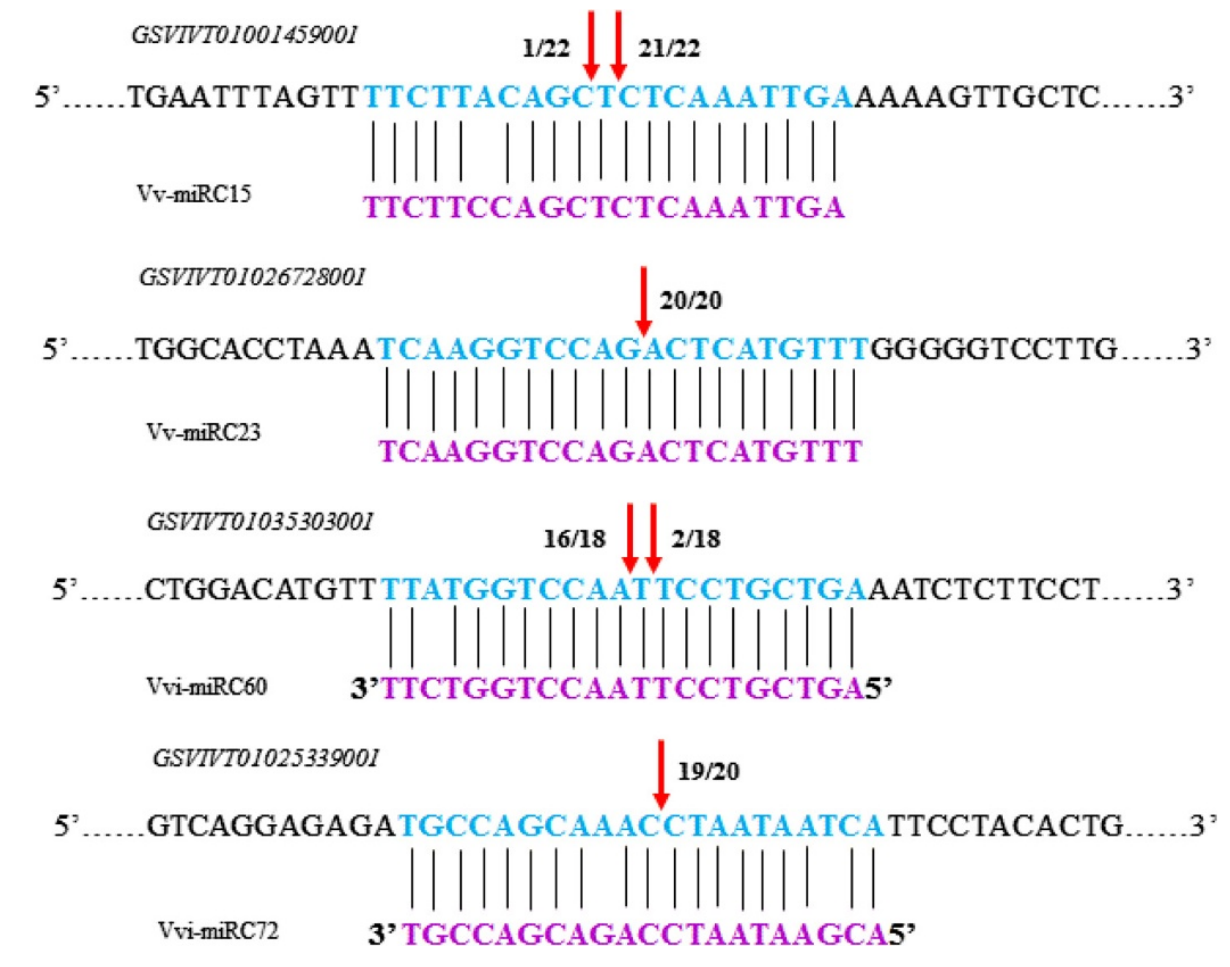

Figure 6 Verification of target genes for Vvi-miRNAs by RLM-5'-RACE. 
grapevine materials used in these studies or $\mathrm{GA}_{3}$ induced/depressed Vvi-miRNAs in this work.

The breakthroughs in sequencing technology have provided the most powerful tool for miRNA discovery, and one of the advantages of the thorough approach is its ability to reveal novel miRNAs. In this work, a large number of novel candidate Vvi-miRNAs were uncovered, with the miRNAs* for some novel Vvi-miRNAs being detected too. The identification of miRNAs*of the candidate miRNAs provides convincing evidence for consideration of these Vvi-miRNAs as authentic [38]. Importantly, the expression levels of some novel Vvi-miRNAs and their miRNAs* under $\mathrm{GA}_{3}$ treatments had much more diverse variation compared to the control. The best examples of this phenomenon are Vvi-miRC20/Vvi-miRC20*, Vvi-miRC34/ Vvi-miRC34*, Vvi-miRC52/Vvi-miR C52* and Vvi-miRC53/ Vvi-miRC53* (Figure 7). The expressions of Vvi-miRC20 and Vvi-miRC34 were up-regulated in $\mathrm{GA}_{3}$ treatments compared to the control, while the expression of their miRNAs* was down-regulated. On the contrary, the expressions of Vvi-miRC52 and Vvi-miRC53 were down-regulated in $\mathrm{GA}_{3}$ treatments, while those of their miRNAs* were upregulated (Figure 7). The reasons for these discrepancies have not been clearly elucidated. The drastic variation in expression levels of the Vvi-miRNA* under $\mathrm{GA}_{3}$ treatments could indicate these miRNAs* were important regulatory genes like Vvi-miRNAs [41].

From high throughput sequencing and qRT-PCR analysis, it was further revealed that the conserve Vvi-miRNAs responsive to $\mathrm{GA}_{3}$ had the higher consistency between these two assays, while the novel ones showed some distinct discrepancy. This might be derived from the fact that the conserved Vvi-miRNAs possessed the higher conservation of development and function, while the novel ones had high specificity. Other, the samples used in the high throughput sequencing were the mixed materials from grapevine berries of several development stages, while those used for the qRT-PCR were of three stages berries (1 week after flowering (WAF), 5 WAF, 9 WAF). These could be the reasons explaining the expression levels of novel VvimiRNAs from qRT-PCR and high throughput sequencing had more apparent differences than those of conserved Vvi-miRNAs. In addition, the predication of potential target genes for novel Vvi-miRNAs responsive to exogenous $\mathrm{GA}_{3}$ and functional annotations of their orthologous target genes in other plants revealed that 20 genes were related to stress resistance. Whether or how these $\mathrm{GA}_{3}$ responsive novel Vvi-miRNAs are involved in the regulation of stress

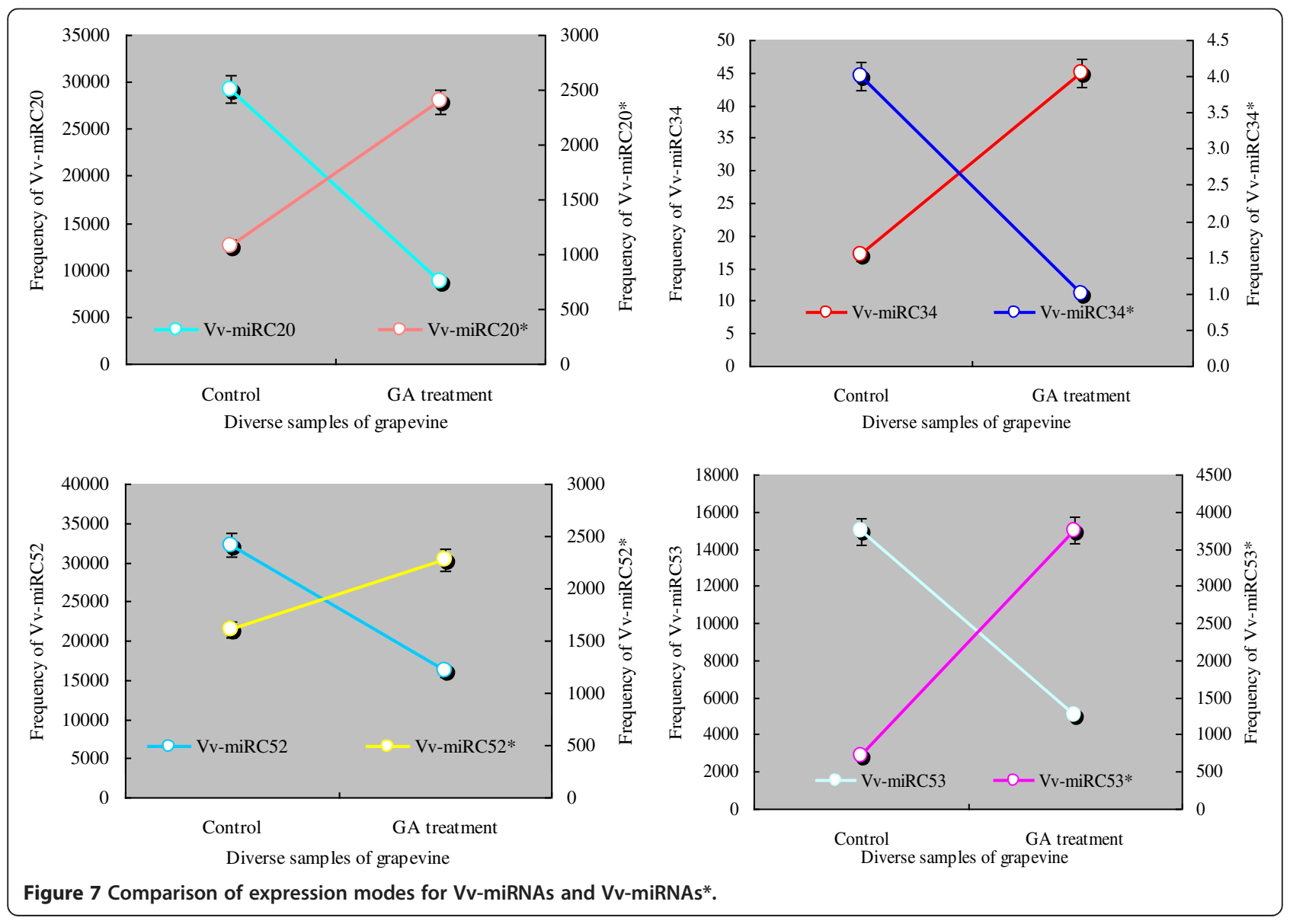


resistance can call for further research. The functions of one-third of the novel Vvi-miRNAs were unknown, indicating that more studies need to be performed on these novel miRNAs to elucidate their functions in the growth of grapevine.

\section{Conclusions}

Deep sequencing of short RNAs from grapevine berries in GA3 treatment and the control identified $137 \mathrm{GA}_{3}$-responsive miRNAs, of which 28 exhibited different expression profiles of response to $\mathrm{GA}_{3}$ in the diverse developmental stages of grapevine berries. These Vvi-miRNAs identified might be involved in the grapevine berry development and response to various environments.

\section{Methods}

\section{Plant materia}

Mixed samples of young berries (one week after flowering, WAF1) large berries (five week after flowering, WAF5), and old berries(nine week after flowering, WAF9) treated with $50 \mathrm{mg} / \mathrm{lGA}_{3}$, were collected in 2011 from four-year old 'Summer Black' grapevine (hybrids of $V$. vinifera and $V$. labrusca) trees grown under common field conditions at the Nanjing Agricultural University fruit farm, Nanjing, China. Each type of samples had three replicates during deep sequencing and qRT-PCR. All the samples were immediately frozen in liquid nitrogen and stored at $-80^{\circ} \mathrm{C}$ until use.

\section{Small RNA library construction and sequencing}

Mixed Summer Black' grapevine young berries (one week after flowering), large berries (five week after flowering after flowering), and old berries (nine week after flowering) treated with GA3, wereusedforRNAextraction. The total RNA samples were first extracted usingour modified CTAB method [20]. Isolation of small RNAs and preparation of small RNA libraries were performed based on the procedure of Wang et al. (2011) [20]. sRNAs were first separated from the total RNA by size fractionation with 15\% TBE urea polyacrylamide gel (TBU) and small RNA regions correspondingto the 18-30 nucleotide bands in the marker lane were excised and recovered. The 18-30 nt small RNAs were 5' and 3' RNA adapter-ligated by T4 RNA ligase and at each step, length validated and purified by TBU electrophoretic separation. The adapter-ligated smallRNA was subsequently transcribed into cDNA by SuperScript II Reverse Transcriptase (Invitrogen) and PCR amplified using primers that annealed to the ends of the adapters. The amplified cDNA constructs were purified and recovered. $18 \mathrm{ng}$ cDNA was loaded into the Illumina $1 \mathrm{G}$ Genome Analyzer for sequencing.

\section{Bioinformatics analysis and identification of Vvi-miRNA} To identify conserved and potential Vvi-miRNAs in grapevine, the raw sequences were processed as described by
Sunkaret al. (2005) [8]. All sRNAs sequences from 18nt to 30nt obtained were removed from the vector sequences, then the modified sequences were further subjected to removal of rRNA, tRNA, snRNA, snoRNA and those containing the polyA tails, and finally the remaining sequences were compared against known plant Vvi-miRNAs in the miRBase [33]. Only the high matching (0-3mismatches) sequences were considered as conserved Vvi-miRNAs. To study potential Vvi-miRNAs precursor sequences, all sRNAs from grapevine were aligned against the grapevine genome and then the miRNA candidates were processed by miRCat (http://srna-tools.cmp.uea.ac.uk/) [33], using default parameters, to generate the secondary structures (Additional file 1).

\section{qRT-PCR validation of miRNA expression}

The template for RT-PCR was the miRNA-enriched library mentioned above. To amplify the Vvi-miRNAs from the reverse transcribed cDNAs, we used the Vvi-miRNA precise sequences as the forward primers and the mirRacer 3'Primer as the reverse primer [9] (Additional file 3). RT-PCR was conducted with the Rotor-Gene 3000 (Corbett Robotics, Australia) and the Rotor-Gene software version 6.1. For each reaction, $1 \mu \mathrm{L}$ of diluted cDNA (equivalent to about $100 \mathrm{pg}$ of total RNA) was mixed with $10 \mu \mathrm{L}$ of $2 \mathrm{X}$ SYBR green reaction mix $\left(\mathrm{SYBR}^{\circ}\right.$ Green qRT-PCR Master Mix; Toyobo, Osaka, Japan), and 5 pmol each of the forward and the reverse primers were added in a final volume of $20 \mu \mathrm{L}$. The conditions for the PCR amplification were as follows: polymerase activation at $95^{\circ} \mathrm{C}$ for $1 \mathrm{~min}$, then $95^{\circ} \mathrm{C}$ for $1 \mathrm{~min}$, followed by 50 cycles of $95^{\circ} \mathrm{C}$ for $15 \mathrm{~s}, 95^{\circ} \mathrm{C}$ for $15 \mathrm{~s}, 60^{\circ} \mathrm{C}$ for $20 \mathrm{~s}$, and $72^{\circ} \mathrm{C}$ for $20 \mathrm{~s}$. The comparative quantification procedure was used to determine relative expression levels, and the homologous genes of the Arabidopsis $5.8 S$ rRNA in grapevine berries was previously used as a reference gene in the qPCR detection of miRNAs in Arabidopsis [41]. The data were analyzed with an $\mathrm{R}^{2}$ above 0.998 using the LinRegPCR program [42].

\section{Prediction of potential target mRNAs for Vvi-miRNAs}

Target predictions were performed based on methods described by Schwab et al.(2005) [39]. Putative Vvi-miRNAs were first blasted against the grapevine unigene database on the Genoscope (http://www.genoscope.cns.fr/). BLASTn hits possessing less than four mismatches were chosen as the candidate targets, and then BLASTx was used to obtain their putative functions. The sequences of predicted targets and their functions are shown in Additional file 4.

\section{Data access}

The sRNA sequence data from this study have been submitted to Gene Expression Omnibus (GEO) under accession No. at website: http://www.ncbi.nlm.nih.gov/geo/ query/acc.cgi?token=dlihnquim uscezm\&acc=GSE3973. 


\section{Additional files}

\section{Additional file 1: Secondary structures of the identified novel} Vv-miRNAs.

Additional file 2: Novel Vv-miRNAs identified in $\mathrm{GA}_{3}$ treated and the control grapevine berries.

Additional file 3: List of predicted target genes of novel miRNAs identified in grapevines.

Additional file 4: Primer sequences of qRT-PCR validated novel miRNAs.

\section{Competing interests}

The authors declare that they have no competing interests.

\section{Authors' contributions}

$\mathrm{JH}$ and $\mathrm{CW}$ carried out the molecular genetic studies, participated in the sequence alignment. YY and XS carried out the RT-PCR arrays. XL and CS participated in the sequence alignment. CW performed the statistical analysis. JH and CW drafted the manuscript. JF and CW conceived of the study, and participated in its design and coordination. JF revised the manuscript. All authors read and approved the final manuscript.

\section{Acknowledgements}

This research was supported by Project Funded by the Natural Science Foundation of China (NSFC) (No. 31301759), China Postdoctoral Science Foundation(2013 M531373), Postdoctoral Science Foundation of Jiangsu Province(1301116C), the Priority Academic Program Development of Jiangsu Higher Education Institutions (PAPD), the National Science Foundation of China (No. 60901053), and the Nanjing Agricultural University Youth Science and Technology Innovation Fund (KJ2013013).

\section{Author details}

${ }^{1}$ College of Horticulture, Nanjing Agricultural University, Nanjing 210095,

China. ${ }^{2}$ Institute of Pomology, Taian 210038, Shandong, China.

Received: 1 July 2013 Accepted: 3 February 2014

Published: 8 February 2014

\section{References}

1. Chen X: A microRNA as a translational repressor of APETALA2 in Arabidopsis flower development. Science 2004, 303:2022-2025.

2. Lauter N, Kampani A, Carlson S, Goebel M: Moose SP: microRNA172 downgulates glossy15 to promote vegetative phase change in maize. Proc Natl Acad Sci USA 2005, 102:9412-9417.

3. $\mathrm{Fu} \mathrm{JH}$, Chiou TJ, Lin Sl, Aung K, Zhu JK: A miRNA involved in phosphatestarvation response in Arabidopsis. Curr Biol. 2005, 15:2038-2043.

4. Huq E: Degradation of negative regulators: a common theme in hormone and light signaling networks? Trend Plant Sci. 2006, 11:4-6.

5. Phillips J, Dalmay T, Bartels D: The role of small RNAs in abiotic stress. FEBS Letters 2007, 581:3592-3597.

6. Carthew RW, Sontheimer EJ: Origins and mechanisms of miRNAs and siRNAs. Cell 2009, 136:642-655.

7. Carra A, Mica E, Gambino G, Pindo M, Moser C, Pe ME, Schubert A: Cloning and characterization of small non-coding RNAs from grape. Plant $J$ 2009, 59:750-763.

8. Sunkar R, Girke T, Jain PK, Zhu JK: Cloning and characterization of microRNAs from rice. Plant Cell 2005, 17:1397-1411.

9. Wang C, Shangguan LF, Nicholas KK, Wang XC, Han J, Song CN, Fang JG Characterization of microRNAs identified in a table grapevine cultivar with validation of computationally predicted grapevine miRNAs by miRRACE. PLoSone 2011, 6:e21259.

10. Allen E, Xie Z, Gustafson AM, Sung GH, Spatafora JW, Carrington JC: Evolution of microRNA genes by inverted duplication of target gene sequences in Arabidopsisthaliana. Nat Genet. 2004, 36:1282-1290.

11. Rajagopalan R, Vaucheret $H$, Trejo J: Bartel DP: A diverse and evolutionarily fluid set of microRNAs in Arabidopsis thaliana. Genes Dev. 2006, 20:3407-3425.

12. Sunkar R, Zhou X, Zheng $Y$, Zhang W, Zhu JK: Identification of novel and candidate $\mathrm{V} v$-miRNAs in rice by high throughput sequencing. BMC Plant Biol. 2008, 8:25.
13. Szittya G, Moxon S, Santos DM, Jing R, Fevereiro MP, Moulton V, Dalmay T: High-throughput sequencing of Medicagotruncatula short RNAs identifies eight new Vv-miRNA families. BMC Genomics 2008, 9:593.

14. Ruan MB, Zhao YT, Meng ZH, Wang XJ, Yang WC: Conserved miRNA analysis in Gossypiumhirsutum through small RNA sequencing. Genomics 2009, 94:263-268.

15. Zhao CZ, Xia H, Frazier TP, Yao YY, Bi YP, Li YP, Li AQ, Li MJ, Zhang BH, Wang $X J$ : Deep sequencing identifies novel and conserved microRNAs in peanuts (Arachishypogaea L.). BMC Plant Biol 2010, 10:3.

16. Moxon S, Jing R, Szittya G, Schwach F, RusholmePilcher RL, Moulton V, Dalmay T: Deep sequencing of tomato short RNAs identifies microRNAs targeting genes involved in fruit ripening. Genome Res 2008, 18:1602-1609.

17. Song CN, Wang C, Zhang CQ, Korir NK, Yu HP, Ma ZQ, Fang JG: Deep sequencing discovery of novel and conserved microRNAs in trifoliate orange (Citrus trifoliata). BMC Genomics 2010, 11:431.

18. Pantaleo V, Szittya G, Moxon S, Miozzi L, Moulton V, Dalmay T, Burgyan J. Identification of grapevine microRNAs and their targets using highthroughput sequencing and degradome analysis. The Plant Journal 2010, 62:960-976.

19. Mica E, Piccolo V, Delledonne M, Ferrarini A, Pezzotti M, Casati C, Fabbro CD, Valle G, Policriti A, Morgante M, Pesole G, Enrico MP, Horner DS: Correction: High throughput approaches reveal splicing of primary microRNA transcripts and tissue specific expression of mature microRNAs in Vitisvinifera. BMC Genomics 2010, 11:109.

20. Wang C, Wang X, Nicholas KK, Song C, Zhang C, Li X, Han J, Fang J: Deep sequencing of grapevine flower and berry short RNA library for discovery of new microRNAs and validation of precise sequences of grapevine microRNAs deposited in miRBase. PhysiologiaPlantarum 2011, 143:64-81.

21. Wang C, Han J, Liu C, Kibet KN, Kayesh E, Shangguan L, Li X, Fang J: Identification of microRNAs from Amur grape (VitisamurensisRupr.) by deep sequencing and analysis of microRNA variations with bioinformatics. BMC Genomics 2012, 13:122.

22. He PC: Grapevine [M]. Beiling: Chinese Agricultural Press; 1998.

23. Leyser O: Plant hormones. Curr. Biol. 1998, 8:R5-R7

24. Klee H: Hormones are in the air. PNAS 2003, 100:10144-10145

25. Abu-Zahra TR: Berry size of Thompson seedless as influenced by the application of Gibberellic acid and can girdling. Pak J Bot 2010, 42:1755-1760.

26. Abu-Zahra TR, Salameh NM: Influence of Gibberellic acid and can girdling on berry size of black magic grape cultivar. Middle-East Journal of Scientific Research 2012, 11:718-722.

27. Achard P, Herr A, Baulcombe DC, Harberd NP: Modulation of floral development by a gibberellin-regulated microRNA. Development 2004 131:3357-3365.

28. Reyes $J$, Chua NH: ABA induction of miR159 controls transcript levels of two MYB factors during Arabidopsis seed germination. Plant Journal 2007 49(4):592-606

29. Gsukasi F, Donaire L, Casanal A, Martinez-Priego L, Botella MA, MedinaEscobar N, Llave C, Valpuesta V: Two strawberry miR159 family members display developmental-specific expression patterns in fruit receptacle and cooperatively regulate Fa-GAMYB. New phytologist 2012, 195:47-57.

30. Jaillon O, Aury JM, Noel B, Policriti A, Clepet C, Casagrande A, Choisne N, Aubourg S, Vitulo N, Jubin C, Vezzi A, Legeai F, Huqueney P, Dasilva C, Horner D, Mica E, Jublot D, Poulain J, Bruyère C, Billault A, Segurens B, Gouyvenoux M, Ugarte E, Cattonaro F, Anthouard V, Vico V, Fabbro CD, Alaux M, Gaspero GD, Dumas V, Felice N, Paillard S, Juman I, Moroldo M, Scalabrin S, Canaguier A, Clainche IL, Malacrida G, Durand E, Pesole G, Laucou V, Chatelet P, Merdinoglu D, Delledonne M, Pezzotti M, Lecharny A, Scarpelli C, Artiguenave F, Pè ME, Valle G, Morgante M, Caboche M, Adam-Blondon AF, Weissenbach J, Quétier F, Wincker P: French-Italian Public Consortium for Grapevine Genome Characterization: the grapevine genome sequence suggests ancestral hexaploidization in major angiosperm phyla. Nature 2007, 449:463-467.

31. Henderson IR, Zhang XY, Lu C, Johnson L, Blake C, Meyers BC, Green PJ, Jacobsen SE: Dissecting Arabidopsis thaliana DICER function in small RNA processing, gene silencing and DNA methylation patterning. Nature Genet. 2006, 38:721-725.

32. Morin RD, Aksay G, Dolgosheina E, Ebhardt HA, Magrini V, Mardis ER, Sahinalp SC, Unrau PJ: Comparative analysis of the small RNA transcriptomes of Pinuscontorta and Oryza sativa. Genome Res. 2008, 18:571-584. 
33. Wang $C$, Leng $X P$, Zhang $Y Y$, Kayesh E, Zhang YP, Sun $X$, Fang JG: Transcriptome-wide analysis of dynamic variations in regulation modes of grapevine microRNAs on their target genes during grapevine development. Plant Molecular Biology 2014, 84(3):269-285.

34. Qiu DY, Pan XP, Wilson IW, Li F, Liu M, Teng W, Zhang B: High throughput sequencing technology reveals that the taxoid elicitor methyl jasmonate regulates microRNA expression in Chinese yew (Taxuschinensis). Gene 2009, 436:37-44.

35. Lindow M, Krogh A: Computational evidence for hundreds of non-conserved plant microRNAs. BMC Genomics 2005, 6:119.

36. Zhang XR, Luo GG, Wang RH, Wang J, Himelrick DG: Growth and developmental responses of seeded and seedless grape berries to shoot girdling. J Am SocHortSci 2003, 128:316-323.

37. Meyers BC, Axtell MJ, Bartel B, Bartel DP, Baulcombe D, Bowman JL, Cao XF, Carrington JC, Chen XM, Green PJ, Griffiths-Jones S, Jacobsen SE, Mallory AC, Martienssen RA, Poethig RS, Qi YJ, Vaucheret H, Voinnet O, Watanabe Y, Weigel D, Zhu JK: Criteria for annotation of plant MicroRNAs. Plant Cell 2008, 20:3186-3190

38. Schwab R, Palatnik JF, Riester M, Schommer C, Schmid M: Weigel D:Specific effects of microRNAs on the plant transcriptome. Dev Cell 2005, 8(4):517-527.

39. Jagadeeswaran GR, Zheng Y, Sumathipala N, Jiang HB, Arrese EL, Soulages JL, Zhang WX, Sunkar R: Deep sequencing of small RNA libraries reveals dynamic regulation of conserved and novel microRNAs and microRNA-stars during silkworm development. BMC Genomics 2010, 11:52.

40. Mallory AC, Bartel DP, Bartel B: MicroRNA-directed regulation of Arabidopsis AUXIN RESPONSE FACTOR17 is essential for proper development and modulates expression of early auxin response genes. Plant Cell 2005, 17:1360-1375.

41. Shi $R$, Chiang VL: Facile means for quantifying microRNA expression by real-time PCR. Biotechniques 2005, 39:519-525.

42. Ramakers C, Ruijter JM, Deprez RH, Moorman AF: Assumption-free analysis of quantitative real-time polymerase chain reaction (PCR) data. Neuroscience Lett 2003, 339:62-66.

doi:10.1186/1471-2164-15-111

Cite this article as: Han et al:: Grapevine microRNAs responsive to exogenous gibberellin. BMC Genomics 2014 15:111.

\section{Submit your next manuscript to BioMed Central and take full advantage of:}

- Convenient online submission

- Thorough peer review

- No space constraints or color figure charges

- Immediate publication on acceptance

- Inclusion in PubMed, CAS, Scopus and Google Scholar

- Research which is freely available for redistribution 